\title{
On the Improvement of Walking Performance in Natural Environments by a Compliant Adaptive Gait
}

\author{
Elena Garcia and Pablo Gonzalez de Santos
}

\begin{abstract}
It is a widespread idea that animal-legged locomotion is better than wheeled locomotion on natural rough terrain. However, the use of legs as a locomotion system for vehicles and robots still has a long way to go before it can compete with wheels and trucks, even on natural ground. This paper aims to solve two main disadvantages plaguing walking robots: their inability to react to external disturbances (which is also a drawback of wheeled robots); and their extreme slowness. Both problems are reduced here by combining: 1) a gait-parameter-adaptation method that maximizes a dynamic energy stability margin and 2) an active-compliance controller with a new term that compensates for stability variations, thus helping the robot react stably in the face of disturbances. As a result, the combined gait-adaptation approach helps the robot achieve faster, more stable compliant motions than conventional controllers. Experiments performed with the SILO4 quadruped robot show a relevant improvement in the walking gait.
\end{abstract}

Index Terms-Compliance control, gait adaptation, legged locomotion, stability criteria.

\section{INTRODUCTION}

$\mathbf{I}$ T HAS BEEN thought that one day walking robots will replace wheeled machines on natural uneven terrain, yet there are still virtually no real walking robots robust enough to walk successfully in natural environments. In spite of the initial expectations, most walking robots are still laboratory prototypes, and their industrial application is still far from occurring. One can find extensive work in the literature on the design and control of statically stable gaits that are good enough for walking on rough terrain [1]-[3]. Although these algorithms can help the robot negotiate rough terrain, they utterly ignore the question of adapting to the disturbing effect of intrinsic robot dynamics, on-board manipulator dynamics, or environmental disturbances.

Previous work on adaptive dynamic walking is biologically inspired, based on neural oscillators [4]-[7]. Among them, only the work by Kimura et al. [7] achieved dynamic walking over medium-rough natural terrain. Furthermore, the performance of

Manuscript received April 20, 2006. This paper was recommended for publication by Associate Editor H. R. Choi and Editor H. Arai upon evaluation of the reviewers' comments. This work was supported by the Spanish Ministry of Education and Science under Grant DPI2004-05824. The work of E. Garcia was supported by the European Social Fund under Postdoctoral Contract CSIC-I3P. This paper was presented in part at the 8th International Conference on Climbing and Walking Robots, London, U.K., September 2005.

The authors are with the Industrial Automation Institute-CSIC, La Poveda, 28500 Madrid, Spain (e-mail: egarcia@iai.csic.es).

This paper has supplementary multimedia material available at http://ieeexplore.ieee.org, provided by the author. This material consists of five videos in mpg format, showing the experiments discussed in this paper.

Digital Object Identifier 10.1109/TRO.2006.884343 systems controlled by central pattern generators (CPGs) can potentially suffer if significant disturbances exist [8]. In fact, none of the biologically inspired work deals with external disturbances during the gait.

Active-compliance controllers have been used to control walking-robot gaits successfully [9]-[11]. This kind of controller produces a motion similar to that produced by a spring following Hook's law, connecting a foot directly to the body. This helps the robot recover from small perturbations. However, when walking on inclined ground or troubled by sizeable disturbances, the transient response of the compliant motion can itself cause instability.

In this paper, we propose to improve the robustness of quadrupedal walking on uneven terrain via a method that enables the robot to recover from external disturbances and increase average gait speed. The gait is based on a classical two-phase discontinuous gait [1] of the type widely used in statically stable walking machines. The gait is automatically adapted to uneven terrain and external forces and moments by a novel gait-parameter adaptation algorithm, while a body-regulation system based on active compliance helps the robot react to impulsive disturbances. And we move one step beyond at this point by improving the robustness of the active-compliance controller by inserting a new term in the active-compliance equation that is proportional to the variation of a dynamic stability margin. As a result, the proposed adaptive and compliant gait improves machine speed and stability in the presence of internal perturbations (caused by robot dynamics) and external perturbations (caused by the environment). The dynamic stability margin used in this paper is the normalized dynamic energy stability margin, or $S_{\text {NDESM }}$ [12], which quantifies the impact energy that a robot can withstand. This stability margin was chosen in the light of recent results on the classification of stability margins, which has shown that the most accurate stability margin when robot dynamics are significant is $S_{\mathrm{NDESM}}$ [13]. This stability measurement is used throughout this paper to demonstrate the efficiency of the proposed approach in experiments with a real robot. Computation of $S_{\text {NDESM }}$ is detailed in the Appendix. Videos of every experiment described in this paper are available at $\mathrm{http}: / /$ ieeexplore.ieee.org.

The outline of the paper is as follows. The problem approach is described in Section II. The proposed dual method for achieving a compliant adaptive gait is described in Section III. The experimental setup is detailed in Section IV, which includes testbed description, signal processing, and tuning of controller gains. Experiments showing the improvement afforded by the proposed method are reported in Section V. Finally, some conclusions are presented. 


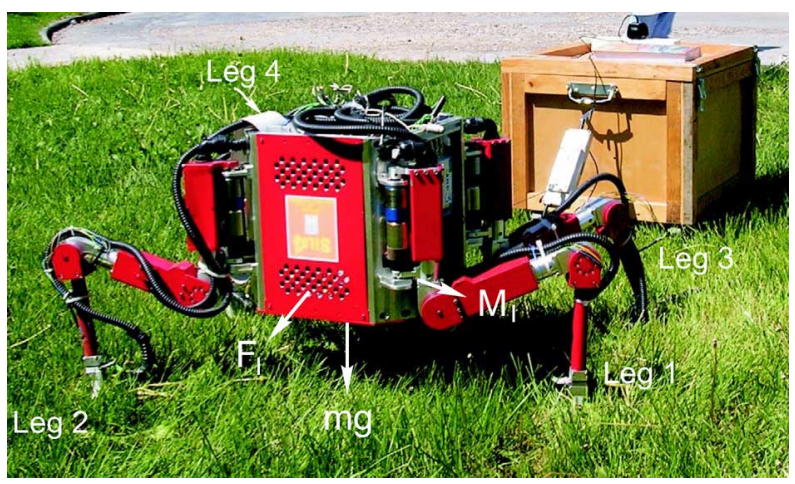

Fig. 1. Quadruped robot SILO4 walking on natural terrain pulling a load. Resultant of inertial forces $\mathbf{F}_{\mathbf{I}}$ and moments $\mathbf{M}_{\mathbf{I}}$ and gravitational forces $\mathbf{m g}$ have been plotted. (Color version of Fig. 1 is available online at http://ieeexplore.org.)

\section{Problem APPROACH}

Let us consider a quadruped robot during its motion on natural terrain, where inertial forces $\mathbf{F}_{\mathbf{I}}$ and moments $\mathbf{M}_{\mathbf{I}}$ due to leg transfer and body motion become significant (see Fig. 1). While in a natural environment, the robot is subject to the impact of external disturbances such as a gust of wind, or unforeseen contact with the surroundings, that added to the robot's internal dynamics, may cause instability. Furthermore, the quadruped robot walks on uneven ground, which implies a nonhorizontal support polygon and a reduction of the stability margin. The robot walks in a two-phase discontinuous gait, while gait stability is controlled using a dynamic-stability criterion.

Following the dynamic-stability criterion, a quadruped is dynamically stable if every moment $M_{i}$ around the $i$-edge of the support polygon due to robot/ground forces and moments is positive, with the vector that goes around the support polygon in the clockwise direction being positive, that is

$$
M_{i}>0, \quad i=1, \ldots, n-1
$$

where $i$ is the edge of the support polygon, and $n$ is the number of supporting feet. $M_{i}$ is the moment around the axis $i$, and comes from

$$
M_{i}=\left(\left(\mathbf{F}_{\mathbf{I}}+\mathbf{m g}\right) \times \mathbf{R}+\mathbf{M}_{\mathbf{I}}\right) \cdot \mathbf{e}_{\mathbf{i}} .
$$

If (1) is true, the robot is dynamically stable, and then the NDESM is defined as the smallest of the stability levels required to tumble the robot around the support polygon, normalized to the robot mass, that is

$$
S_{\mathrm{NDESM}}=\frac{\min \left(E_{i}\right)}{m g}
$$

where $E_{i}$ stands for the stability level of the $i$ th side of the support polygon, which physically means the increment of mechanical energy required to tumble the robot around the $i$ th side of the support polygon, computed from (see Fig. 2)

$$
\begin{aligned}
E_{i}=m g|\mathbf{R}|(\cos \phi & -\cos \varphi) \cos \Psi \\
& +\left(\mathbf{F}_{\mathbf{I}} \cdot \mathbf{t}\right)|\mathbf{R}| \theta+\left(\mathbf{M}_{\mathbf{I}} \cdot \mathbf{e}_{\mathbf{i}}\right) \theta-\frac{1}{2} I_{i} \omega_{i}^{2}
\end{aligned}
$$

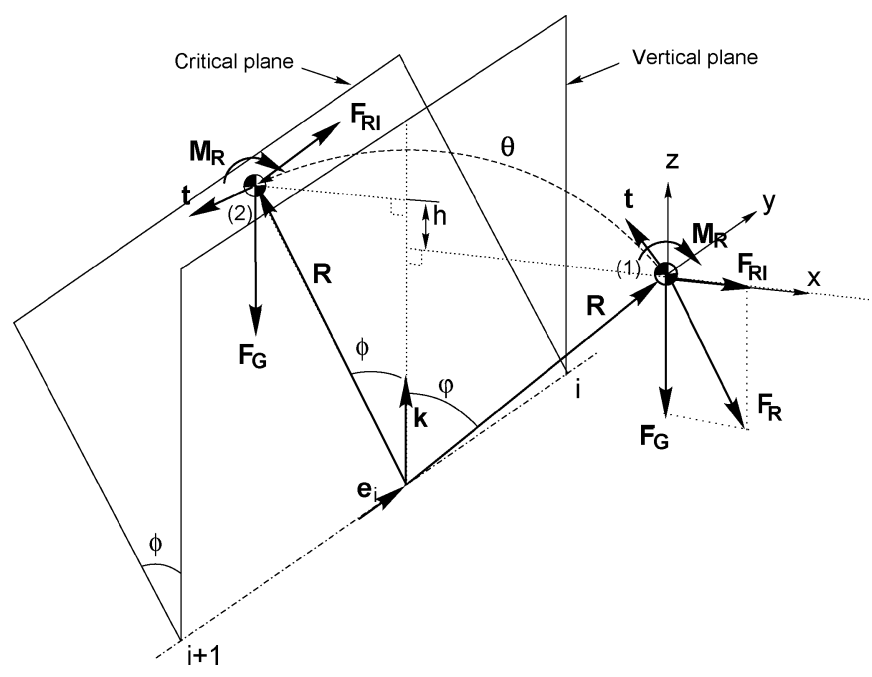

Fig. 2. Outline of the $S_{\text {NDESM }}$ computation.

where $\mathbf{R}$ is a vector orthogonal to the $i$ th side of the support polygon that points to the center-of-gravity $(\mathrm{CG})$ position, $I_{i}$ is the moment of inertia around the rotation axis $i, \omega_{i}$ is the angular velocity of the CG, $\Psi$ is the inclination angle of the $i$ th side of the support polygon, and $\phi, \varphi$, and $\theta$ are the rotation angles around the $i$ axis. $\varphi$ is the rotation angle required to position the CG inside the vertical plane (see Fig. 2); $\phi$ is the angle that the CG rotates from the vertical plane to the critical plane, where the resultant moment acting on the CG vanishes. Finally, $\theta$ is the addition of both rotations. Unitary vector $\mathbf{t}$ is tangent to the CG trajectory, and $\mathbf{e}_{\mathbf{i}}$ is the unitary vector that goes around the support polygon in the clockwise direction. The first three terms on the right side of (4) represent the potential energy required for the tumble caused by gravitational and nongravitational forces and moments, while the fourth term represents kinetic energy (see the Appendix and [12] for a more detailed explanation). $S_{\text {NDESM }}$ is the only dynamic stability margin that considers the effect of impulsive, external disturbances on robot stability.

To show the disturbance that an external force causes in robot stability, an experiment has been carried out using the SILO4 quadruped robot (see Fig. 1) [14], [15]. A digital video of this experiment is available at http://ieeexplore.ieee.org (video1). In this experiment, the SILO4 walking robot performs a two-phase discontinuous gait in natural terrain while it pulls a 50-N load. Additionally, $S_{\text {NDESM }}$ is computed in real time. The SILO4 robot walks during almost two gait cycles at $16 \mathrm{~mm} / \mathrm{s}$, until it turns over due to the disturbance. $S_{\mathrm{NDESM}}$ during the experiment is plotted in Fig. 3. The figure shows the time evolution of the stability margin during the gait which consists of the rear-right leg transfer phase (4), followed by the front-right transfer phase (2), and afterwards body propulsion with all four legs in the support phase (body). The second half of the gait cycle is symmetrical for the legs on the left (shown by the sequence (3)-(1)-(body) in Fig. 3). The stability margin decreases significantly during each leg's transfer phase, especially at the moment of foot lift. The leg inertia at that moment is significant enough to reduce the stability margin, bringing it too close to zero in some phases. The effect of the load disturbance can be observed in the reduction of the stability margin for each phase 


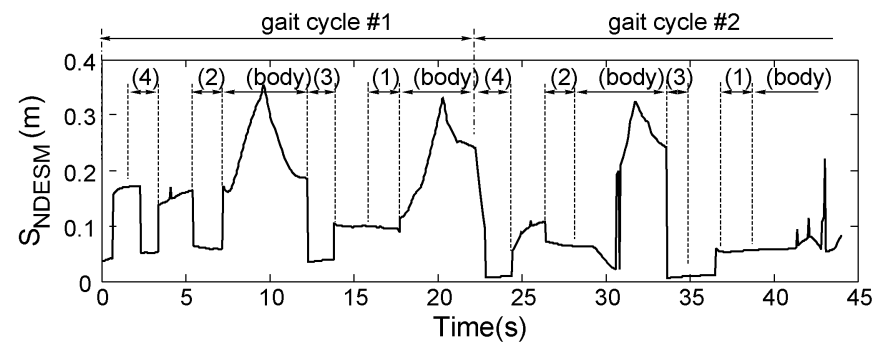

Fig. 3. Time evolution of $S_{\text {NDESM }}$ during the experiment. The leg in transfer phase is shown in brackets, as well as the period of body motion in each gait cycle.

over time. For instance, looking at the stability margin during the transfer phase of leg 4 in the first gait cycle, and comparing it with the stability margin of the same leg transfer phase in the second gait cycle, one can observe a significant decrease in $S_{\text {NDESM }}$ during the second gait cycle. The same $S_{\text {NDESM }}$ reduction can be observed for all other gait phases. The figure and the video show how the robot does not start pulling the load until the first body motion of the first gait cycle. In the second half of the first gait cycle, the robot is affected by a constant disturbance while it transfers legs 3 and 1 . The effect of the constant external disturbance can be noted in the very different stability margins for the transfer phases of these legs. If there is no external disturbance (as during the transfer phase of legs 4 and 2 in the first gait cycle), the stability margins of both legs on a given side (left or right) should be the same on flat, even terrain. If there is a disturbance, the stability margin goes down for the transfer phases of the legs that are closer to the direction of the external force (the rear legs, in this experiment). During the second gait cycle, the stability margin gets distorted, and the robot tumbles down during the last body motion.

To improve the gait's robustness to external disturbances, two steps are required: first, enable the stability margins of the rear and front legs to be leveled in the presence of disturbances, so that the probabilities of tumbling during each leg's transfer phase become equal, and second, compensate for intrinsic leg dynamics to increase the stability margin at leg lifting. As a result, the gait stability margin, which is the smallest stability margin in a gait cycle, will be increased, and this makes for an increase in average gait speed, improving overall walking performance in terms of speed and stability in natural environments.

\section{The AdAptive Compliant Gait}

The goal of the proposed approach is to improve the robustness of the walking gait by means of: 1) leveling the stability margins of the rear and front legs in the presence of disturbances and uneven terrain, so that the probabilities of tumbling during each leg's transfer phase become equal; and 2) increasing the gait stability margin during leg-transfer phases to compensate for internal and environmental perturbations. Objective 1) is accomplished by means of a gait-parameter-adaptation approach, herein proposed, that modifies gait parameters to maximize the stability margin during body motion and to level the stability margin during transfer phases. Objective 2) is achieved by means of an active-compliance approach, which has been

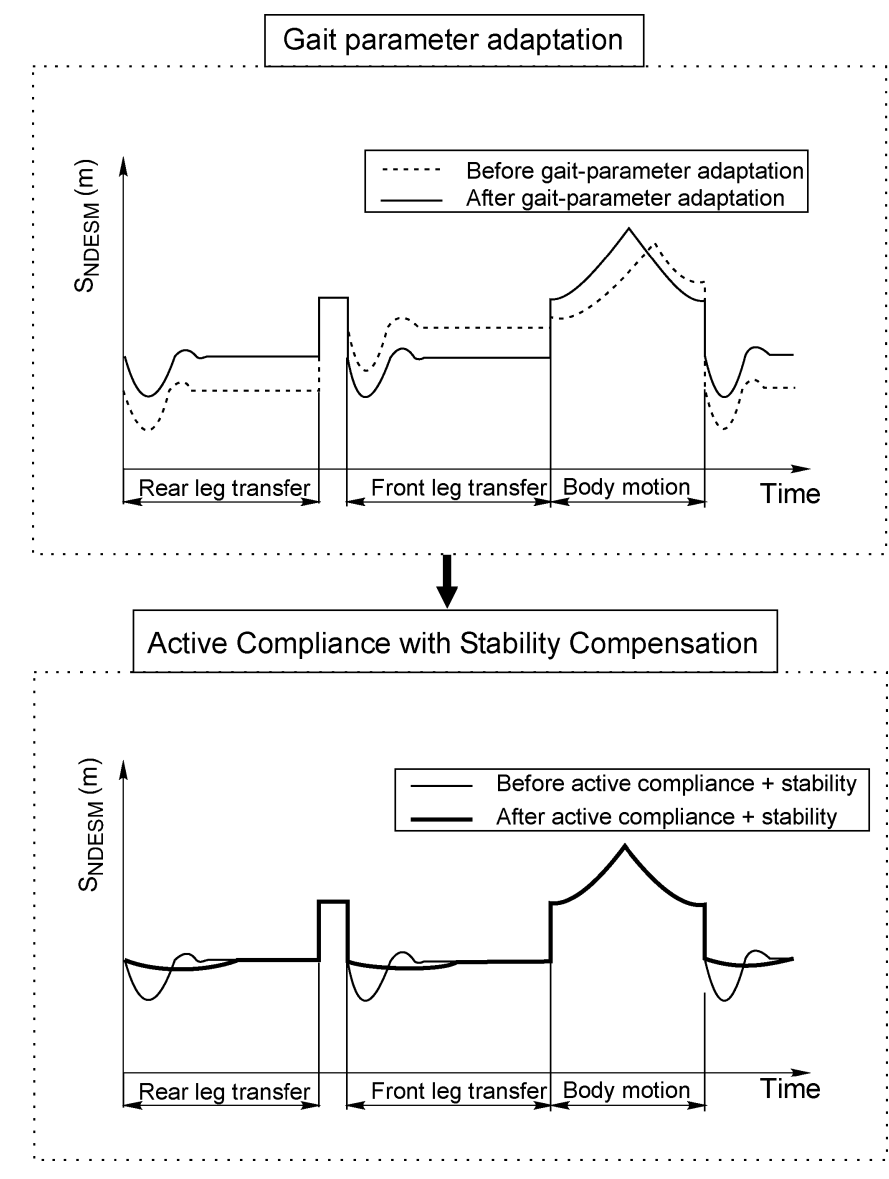

Fig. 4. Proposed gait-adaptation approach in two steps: gait-parameter adaptation to external disturbances and uneven terrain; and active compliance with stability compensation to compensate for internal perturbations. The effect on the stability margin has been drawn for each step.

improved in this paper to compensate for variations in the stability margin during each transfer phase. The proposed dual approach is outlined in Fig. 4, where the effect of each step on the gait stability margin is illustrated. Thus, as the diagram shows, first the gait-parameter-adaptation approach levels the stability margin during the transfer phases and increases the stability margin during body motion. Then, the active-compliance controller with stability compensation herein proposed reduces variations of the stability margin during the transfer phases. As a result of these two steps, the gait stability margin is increased. We will now describe each of these steps, followed by some experiments with SILO4 that show the improvement in gait stability and gait speed.

\section{A. Gait-Parameter Adaptation to the Environment}

Humans and animals adapt their gait to terrain inclination and external forces. Fig. 5 shows how human gait parameters change when a person walks uphill or counteracts an external force. The effect of an inclined terrain or a counteracting force on gait is to reduce both the leg stroke $s$, and the CG height $h$. The leg-stroke reduction implies the modification of footholds in the gait cycle. Another effect is the modification of the horizontal position of the CG. These three parameters are modified to enhance stability. Therefore, the computation of a stability 


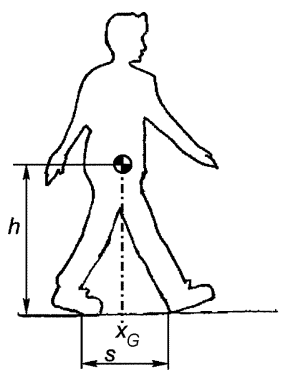

(a)

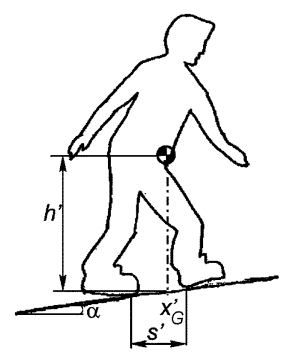

(b)

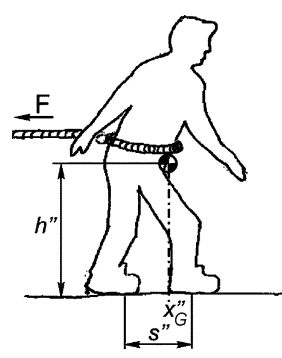

(c)
Fig. 5. CG height $h$ and leg stroke $s$ of a person walking on: (a) flat terrain; (b) inclined terrain; (c) flat terrain under external forces.

margin that considers the destabilizing effect of both ground inclination and external forces, such as $S_{\mathrm{NDESM}}$, is not enough to enhance stability. The gait parameters must also be recomputed to achieve efficient motion control.

Only a few researchers have attempted to partially solve this problem, that involves complex issues such as dynamic modeling and optimization of nonlinear systems. A deliberative planning approach was used in [2] to generate static gaits for the Ambler. This planning approach was used to determine footfalls over uneven terrain, yet it did not consider robot dynamics nor environmental perturbations. A static stability margin was chosen in [16] to maximize gait stability during the transfer phase of a discontinuous gait; however, the stability during the body-motion phase is minimized as a result. Also, because of the consideration of a static-stability margin, robot dynamics are not considered. An optimization method to maximize a dynamic stability margin for a dynamic gait was proposed in [17], yet their work failed to prove that the optimized gait is better when external or manipulation forces appear or the ground is slopped. Recently, the authors of [18] have proposed an automated gait-tuning approach for RHex. This approach is based on parameter optimization, and considers robot dynamics and ground inclination. However, the extremely specific kinematics of the RHex design [with legs of one degree of freedom (DOF)] make it difficult to extend the proposed algorithm to more general leg designs which normally feature three DOFs. A completely different approach was proposed in [5]. They presented a method for tuning CPGs to produce basic locomotion. A static postural reflex succeeded in distributing the robots' weight equally over the feet, so that if the robot was slightly pushed in any direction, the robot counteracted the disturbance. However, the performance of systems controlled by CPGs can potentially suffer if disturbances are significant [8]. Therefore, to these authors' best knowledge, the problem of complete gait adaptation to both robot dynamics and environmental perturbations is still partially unsolved.

The method we propose determines the right gait parameters to adapt the gait to the environment by maximizing stability. For this purpose, foot positions and leg stroke are determined so that $S_{\mathrm{NDESM}}$ is maximum when the robot's CG is placed at the middle of the body-motion phase, and when the leg stroke $s$ is also at its maximum. This condition will make the gait symmetric with respect to the stability margin, as will be explained below. Let us impose a restriction on the problem statement:

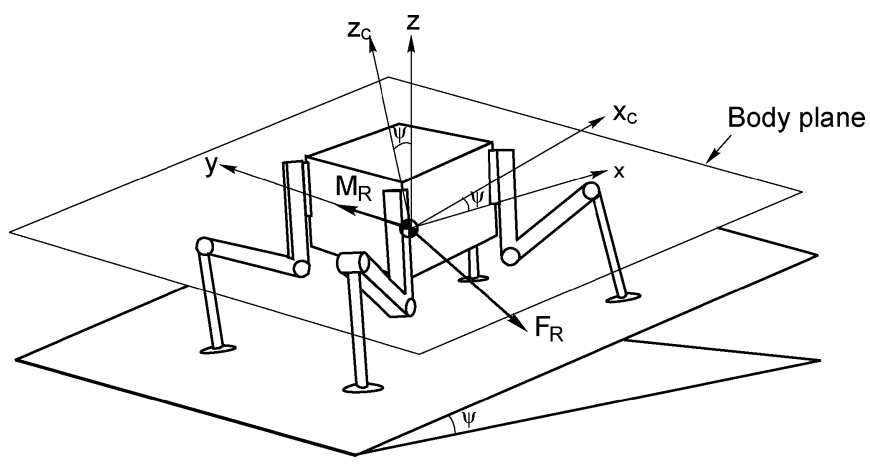

Fig. 6. Quadruped robot on an inclined terrain.

Ground inclination and external forces lie along the robot's longitudinal axis, and this axis coincides with the direction of motion. This restriction is inspired by biological behavior: humans and animals usually align their direction of motion with the direction of the disturbance, to better compensate for it.

The problem statement is as follows.

Let us assume a quadruped robot walking along the direction coincident with its longitudinal axis (see Fig. 6). Let us define an external fixed reference frame $\{x, y, z\}$ and a body-fixed reference frame $\left\{x_{c}, y_{c}, z_{c}\right\}$ centered at the robot's CG so that the robot motion is along the $x_{c}$ axis. Initially, both reference frames are centered at the same point. The terrain can be inclined, forming an angle $\Psi$ with the $x$ axis. No motion along the $y$ axis is allowed. $\mathbf{F}_{\mathbf{R}}$ and $\mathbf{M}_{\mathbf{R}}$ are the resultant robot/ground interaction forces and moments acting on the robot, caused by inertial, elastic, and manipulator-related effects.

The gait parameters have to be determined so that the maximum $S_{\text {NDESM }}$ is reached when the robot stands at the middle of its CG trajectory. This condition will make the gait symmetric with respect to the stability margin. If the resultant gait is symmetric with respect to the stability margin, the probabilities of losing robot stability when a rear leg is lifted or when a front leg is lifted are the same. Once again, nature gave us one more hint: when a person walks uphill, the horizontal position of the person's CG moves slightly forward to compensate for the tangential component of gravity. If the person does not make this change in CG position, he/she will probably tumble downhill at the least unexpected perturbation (like a gust of wind), because the probability of tumbling downhill is larger than uphill. The horizontal motion of the CG will make the probabilities of tumbling downhill and uphill the same, thus decreasing the overall probability of tumbling. Therefore, first of all, $S_{\mathrm{NDESM}}$ has to be expressed in terms of foothold coordinates and CG coordinates. Considering the restriction we have set on the direction of motion, the expression of $S_{\mathrm{NDESM}}$ is simplified to

$$
S_{\mathrm{NDESM}}=\frac{\min \left(E_{i}\right)}{m g}, \quad i=2,4
$$

where $E_{i}$ is computed as

$E_{i}=m g|\mathbf{R}|(\cos \phi-\cos \varphi)+\left(\mathbf{F}_{\mathbf{R I}} \cdot \mathbf{t}\right)|\mathbf{R}| \theta+\left(\mathbf{M}_{\mathbf{R}} \cdot \mathbf{e}_{\mathbf{i}}\right) \theta-\frac{1}{2} I_{i} \omega_{i}^{2}$. 


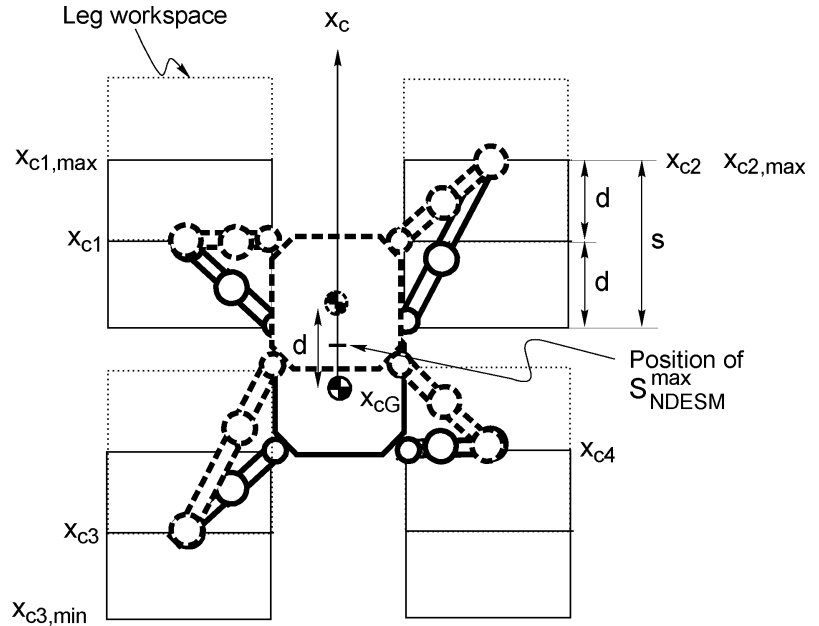

Fig. 7. Outline of gait-parameter adaptation for a quadruped in the body-motion phase of a two-phase discontinuous gait.

Notice that every term in (6) can be expressed as a function of foothold coordinates and CG coordinates in the $\{x, y, z\}$ reference frame, that is

$$
E_{i}=\mathcal{F}\left(x_{i}, y_{i}, z_{i}, x_{i+1}, y_{i+1}, z_{i+1}, x_{G}, y_{G}, z_{G}\right)
$$

where $x_{i}, y_{i}, z_{i}, x_{i+1}, y_{i+1}, z_{i+1}$ are coordinates of two consecutive footholds in the support pattern, and $x_{G}, y_{G}, z_{G}$ are CG coordinates in the fixed reference frame.

The gait-optimization procedure is divided into two phases. The first phase consists of determining foot $x$-positions and the body CG $x$-position along the $x_{c}$ axis, and the leg stroke that optimize the gait; that is, the maximum $S_{\mathrm{NDESM}}$ is reached when the CG is placed at the middle of the body-motion phase and the leg stroke is maximum. The height of the CG is maintained at a value that allows obstacle avoidance during body motion. If the CG height is included in the optimization procedure at this phase, the result will require the robot to walk at a zero CG height, where $S_{\text {NDESM }}$ is maximum. However, this result is absurd for any applications requiring walking robots to negotiate terrain irregularities. Therefore, once the rest of the gait parameters have been obtained for a conservative CG height in the first phase of the gait-optimization procedure, a second phase is carried out to adapt body height to the terrain profile and also enhance stability.

1) First Phase: Leg-Stroke and Foothold Calculation: This phase determines the foot and body CG $x$-positions along the $x_{c}$ axis and the leg stroke that optimize the gait in terms of stability.

Let us use the name $x_{c i}$ for the $x_{c}$-coordinate of foothold $i$ referring to the body frame $\left\{x_{c}, y_{c}, z_{c}\right\}$ just before the bodymotion phase starts. Fig. 7 outlines an overhead view of leg workspaces, footholds, and leg stroke for a quadruped during the body-motion phase in a two-phase discontinuous gait. The four legs are in support, thus propelling the body forward. The initial position of the robot has been plotted in a thick solid line, while the final position of the robot has been plotted in a thick dashed line. $x_{c 1}$ denotes the left front leg's foothold, $x_{c 2}$ denotes the right front leg's foothold, $x_{c 3}$ denotes the left rear leg's foothold, and $x_{c 4}$ denotes the right rear leg's foothold. The CG trajectory has been also plotted, where $x_{c G}$ denotes the $x_{c}$-coordinate of the CG position, and the maximum-stability position lies at the middle of the trajectory. Notice that the length of each body motion is half the leg stroke, that is, $s=2 d$. Therefore, maximizing $s$ is accomplished by maximizing $d$.

The leg stroke can be expressed as a function of the front legs' footholds as follows:

$$
d\left(x_{c 1}, x_{c 2}\right)=x_{c 2}-x_{c 1} \text {. }
$$

The two rear legs' footholds can be expressed in terms of the two front legs' footholds:

$$
\begin{aligned}
& x_{c 3}=X_{c 3_{\text {min }}}+x_{c 2}-x_{c 1} \\
& x_{c 4}=X_{c 3_{\text {min }}}+2 x_{c 2}-2 x_{c 1} .
\end{aligned}
$$

where $X_{c 3_{\min }}$ is the minimum $x_{c}$ position that leg 3 can achieve, due to workspace limitations.

To obtain the optimized gait, $x_{c 1}, x_{c 2}$, and $x_{c G}$ have to be obtained so that $S_{\text {NDESM }}$ is maximum at the middle of the CG trajectory. As foot and body CG positions along the $x_{c}$ axis are required to solve this problem, $S_{\mathrm{NDESM}}$ has to be expressed as a function of footholds and CG position in the body reference frame, that is

$$
S_{\mathrm{NDESM}}=\mathcal{F}\left(x_{c 1}, x_{c 2}, x_{c G}, h\right) .
$$

Notice that in the above expression, the $y_{c}$ coordinates have been omitted as variables due to the restriction set on the direction of motion. The $z_{c}$ coordinates are missing as well, because the robot walks along the $x_{c}$ axis, and every $z_{c}$ component is a function of body CG height, which has been denoted as $h$. Whereas in this first phase of the optimization process, the CG height is considered constant, $h=h_{0}$, so (11) will not depend on the height parameter, and

$$
S_{\mathrm{NDESM}}=\mathcal{F}\left(x_{c 1}, x_{c 2}, x_{c G}\right) .
$$

However, $S_{\mathrm{NDESM}}$ has been expressed in (7) in an external reference frame $\{x, y, z\}$. Therefore, mapping is required from the external reference frame $\{x, y, z\}$ to the body's reference frame $\left\{x_{c}, y_{c}, z_{c}\right\}$. Let us say $\mathbf{G}$ is the position vector of the body reference frame in the external reference frame, that is

$$
\mathbf{G}=\left(x_{G} y_{G} z_{G}\right)^{T} .
$$

Note that $\mathbf{G}$ also matches the current position of the robot's CG in the external reference frame. Now, any point $\mathbf{p}_{\mathbf{c}}$ in the body's reference frame $\left\{x_{c}, y_{c}, z_{c}\right\}$ can be mapped onto the external reference frame by means of the following spatial transformation:

$$
\mathbf{p}=\mathbf{G}+\mathbf{R}_{y}(\Psi) \mathbf{p}_{\mathbf{c}}
$$

where $\Psi$ is the angle between the $x_{c}$ and $x$ axes (see Fig. 6), and $\mathbf{R}_{y}(\Psi)$ is the rotation matrix of an angle $\Psi$ about axis $y$. 
To solve (12), $S_{\mathrm{NDESM}}$ has to be expressed in terms of foothold and CG coordinates, and later mapped onto the body reference frame through (14). As a result, $S_{\mathrm{NDESM}}$ will be expressed in terms of $x_{c}$ coordinates. The analytic solution of (12) yields a complex expression that for the sake of clarity has been solved numerically.

$S_{\mathrm{NDESM}}$ has to reach its maximum when the body CG is located at the middle of the body trajectory; therefore, the objective function is

$$
J_{1}=\mathcal{F}\left(x_{c 1}, x_{c 2}, x_{c G}+\frac{d}{2}\right) .
$$

However, there is also another objective function, because the leg stroke should be maximum, too

$$
J_{2}=d\left(x_{c 1}, x_{c 2}\right)
$$

with the following constraints:

$$
\begin{aligned}
x_{c 1} & \leq X_{c 1_{\max }} \\
x_{c 2} & \leq X_{c 2_{c \max }} \\
4 x_{c 1}-3 x_{c 2} & \geq \xi_{1}-X_{c 1_{\max }}
\end{aligned}
$$

where $X_{c 1_{\max }}$ and $X_{c 2_{\max }}$ are the maximum $x_{c}$ positions that legs 1 and 2 (respectively) can achieve, due to workspace limitations. Thus, the first two constraints (17) and (18) are given by the leg-workspace limits, while the third constraint (19) avoids leg-workspace overlapping, where $\xi_{1} \geq 0$ is a constant. For the SILO4 robot $\xi_{1}=0.1 \mathrm{~m}$ to avoid the feet stepping over each other.

To solve the multiobjective problem, the $\varepsilon$-constraint method is used [19]. This method involves optimizing a primary objective $J_{2}$, and expressing the other objectives in the form of inequality constraints $\left(J_{1} \leq \varepsilon\right)$. Therefore, one of the two objective functions is expressed as a nonlinear constraint. In this case, the primary objective function is (16), and the secondary objective function (15) is expressed as

$$
\mathcal{F}\left(x_{c 1}, x_{c 2}, x_{c G}+\frac{d}{2}\right)-S_{\mathrm{NDESM}}^{\max }<\xi_{2}
$$

where $S_{\mathrm{NDESM}}^{\max }$ is the maximum value of $S_{\mathrm{NDESM}}$ for the current gait configuration. Whenever the gait configuration is changed by the optimization method, the value of $S_{\mathrm{NDESM}}^{\max }$ changes, too.

Therefore, the multiobjective optimization problem is thus reduced to a simple constrained optimization problem, where (16) is the function to maximize, subject to constraints (17)-(20). The problem has been solved numerically for the SILO4 robot by an iterative process, starting from an initial estimate of foot positions $x_{1}$ and $x_{2}$. In each iteration step, the resulting foot positions and body CG position are computed, and the value of $S_{\mathrm{NDESM}}^{\max }$ is computed from (5) for the resulting gait configuration. The suitable selection of $\xi_{2}$ is critical for ensuring a feasible solution.

2) Second Phase: CG-Height Calculation: Once the foot positions and leg stroke have been determined, the second phase tunes the CG height to enhance stability. When, as a result of the first phase of the optimization approach, the leg stroke is reduced in order to adapt the walk to stepped ground or an external force and enhance stability during the body-motion phase, stability is reduced during the transfer phase. This consequently reduces the gait stability margin, which is an undesirable effect. This problem can be solved by reducing the CG height (as mentioned above in this section). To solve this problem, the following condition is stated:

$$
\mathcal{F}(h)-S_{\mathrm{NDESM}}^{0}=0
$$

where $S_{\mathrm{NDESM}}^{0}$ stands for a constant value of the gait stability margin in nominal conditions, that is, on flat terrain when there are no external disturbances. Therefore, $S_{\mathrm{NDESM}}$ is constrained to equal the robot's $S_{\mathrm{NDESM}}$ in the absence of external forces and on flat ground during the transfer phase. The function $\mathcal{F}(h)$ is the result of the first phase of the optimization procedure, where every gait parameter has been obtained. Therefore, the only variable at this phase is CG height $(h)$. This problem is a nonlinear equation of a single variable that has been solved by least-squares optimization.

\section{B. Body Regulation by Active Compliance With Stability Compensation}

Once the gait parameters have been adapted to the environment, the stability margins during the rear- and front-leg transfer phases will be equal. However, the gait stability margin is still reduced during each leg transfer phase, due to internal leg dynamics that are significant at leg lift and foot placement. This second step in the gait-adaptation approach is aimed at increasing the gait stability margin, as well as reacting to perturbations of this sort via body regulation (see Fig. 4). To achieve this goal, we propose to use an active compliance approach with a new term that compensates for $S_{\text {NDESM }}$ variations.

The principle of active compliance in a walking robot consists in controlling the motion of each leg in the support phase so that steady-state force errors at the foot are considered linearly proportional to displacement errors in accordance with Hook's law [9], [10]. However, active compliance can also be implemented with a velocity servo system [11]. Thus, foot-force errors are converted into foot-velocity errors and later mapped to desired joint speed, so the active compliance equation for each supporting leg has the form

$$
\dot{\mathbf{q}}_{c}=\mathbf{J}^{-1}\left[\mathbf{K}_{\mathbf{p}}\left(\mathbf{X}_{\mathrm{des}}-\mathbf{X}\right)+\mathbf{K}_{\mathbf{f}}\left(\mathbf{F}_{\mathrm{des}}-\mathbf{F}\right)+\dot{\mathbf{X}}_{\mathrm{des}}\right]
$$

where $\dot{\mathbf{q}}_{c}$ is a vector of commanded joint speeds, $\mathbf{X}_{\text {des }}$ and $\dot{\mathbf{X}}_{\text {des }}$ are vectors of desired Cartesian foot position and velocity, determined by gait generation, and $\mathbf{F}_{\text {des }}$ is the vector of desired Cartesian foot forces, obtained from the force-distribution algorithm [20]. $\mathbf{X}$ and $\mathbf{F}$ are measured Cartesian foot positions and forces. $\mathbf{J}$ is the Jacobian matrix that transforms Cartesian foot speed into joint speed. $\mathbf{K}_{\mathbf{p}}$ and $\mathbf{K}_{\mathbf{f}}$ are diagonal matrices of gains.

In order to understand the behaviour of the system, let us express (22) in Cartesian workspace coordinates

$$
\dot{\mathbf{X}}_{c}=\dot{\mathbf{X}}_{\mathrm{des}}+\mathbf{K}_{\mathbf{p}}\left(\mathbf{X}_{\mathrm{des}}-\mathbf{X}\right)+\mathbf{K}_{\mathbf{f}}\left(\mathbf{F}_{\mathrm{des}}-\mathbf{F}\right)
$$




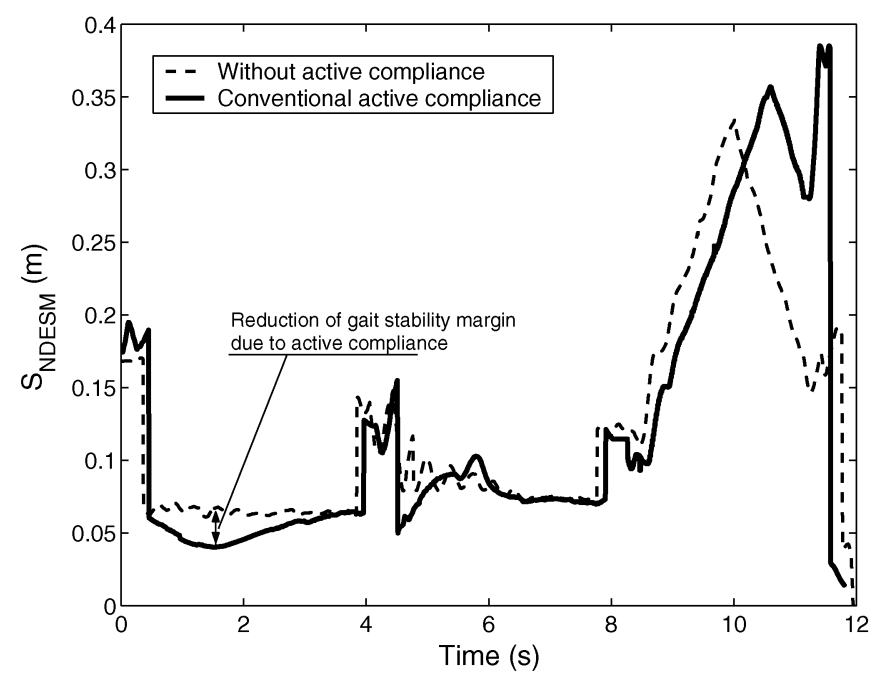

Fig. 8. Reduction of gait stability margin when conventional active compliance is used for body regulation in the SILO4 robot.

which can also be written as

$$
\dot{\mathbf{X}}_{c}=\dot{\mathbf{X}}_{\mathrm{des}}+\delta \dot{\mathbf{X}}
$$

where $\dot{\mathbf{X}}_{c}$ is the vector of commanded foot speeds and

$$
\delta \dot{\mathbf{X}}=\mathbf{K}_{\mathbf{p}}\left(\mathbf{X}_{\text {des }}-\mathbf{X}\right)+\mathbf{K}_{\mathbf{f}}\left(\mathbf{F}_{\text {des }}-\mathbf{F}\right) .
$$

From (24) and (25), we can understand the behaviour of the system: the actual commanded velocities differ from the velocities imposed by the walking gait $\dot{\mathbf{X}}_{\text {des }}$ by a term $\delta \dot{\mathbf{X}}$ that is a tradeoff between position and force errors at the feet. In fact, $\delta \dot{\mathbf{X}}$ is the term that imposes the damped-compliant nature to the system. If we assume that the velocity controller is nearly perfect and that the actual foot speeds $\dot{\mathbf{X}}$ are very close to the commanded speeds $\dot{\mathbf{X}}_{c}$, then we can rearrange (23) as

$$
\mathbf{F}_{\mathrm{des}}-\mathbf{F}=-\mathbf{K}_{\mathbf{p}} \mathbf{K}_{\mathbf{f}}^{-1}\left(\mathbf{X}_{\mathrm{des}}-\mathbf{X}\right)-\mathbf{K}_{\mathbf{f}}^{-1}\left(\dot{\mathbf{X}}_{\mathrm{des}}-\dot{\mathbf{X}}\right)
$$

Equation (26) corresponds to the equation of motion of a massspring-damper system, $\mathbf{K}_{\mathbf{p}} \mathbf{K}_{\mathbf{f}}^{-1}$ being an effective stiffness [reciprocal to effective compliance or compliance ratio (CR)], and $\mathbf{K}_{\mathbf{f}}^{-1}$ the effective damping of the system. It should be noted that there is only one DOF, namely $\mathbf{X}$ motion, to satisfy force, velocity, and position requirements, and that all three cannot be met in a closed kinematic chain. Therefore, a tradeoff among these three magnitudes will be found, which results in the correction of desired foot speeds as a result of position and force feedback.

The above active-compliance controller is used herein to enable the robot to react to internal and external impulsive disturbances during leg-transfer phases. To achieve this, desired ground-reaction forces at each foothold are determined so that the robot's CG stays at zero moments $\mathbf{M}_{\mathbf{x}}$ and $\mathbf{M}_{\mathbf{y}}$. This is a force-distribution problem that has been extensively reported in the literature of force control [9], [20], [21]. However, the compliant motion that helps the robot recover from impulsive disturbances itself reduces the gait stability margin, thus increasing the probability of tumbling down, as Fig. 8 shows. This figure gives a comparison of $S_{\mathrm{NDESM}}$ for the SILO4 robot walking using a position-based controller (dashed line) and conventional active compliance (solid line). As shown, the impulsive perturbation of leg dynamics at leg lift reduces the gait stability margin. Our work deals with this stability reduction, moving one step beyond the current state of the art in active compliance by inserting a term proportional to stability variations in (22). By modifying the robot motion so that stability is increased during leg lifting, we increase the gait stability margin, and therefore, the admissible robot speed. There is one proviso: these CG reaction movements should be in terms of body pitch and roll variations to maintain the gait pattern that was previously adapted to the environment. Therefore, we assume that two angular velocities $\left(\omega_{x}\right.$ and $\left.\omega_{y}\right)$ are desired at the robot's CG during each transfer phase, and that these angular velocities are proportional to the variations of the stability margin with gains $K_{s x}$ and $K_{s y}$, respectively, that is

$$
\begin{aligned}
& \omega_{x}=-K_{s x} \dot{S}_{\mathrm{NDESM}} \\
& \omega_{y}=-K_{s y} \dot{S}_{\mathrm{NDESM}}
\end{aligned}
$$

If we consider that during the leg-transfer phase, the quadruped robot stands on three legs, then the following equation system determines the desired vertical foot speed of each leg in support to generate the desired angular speeds in the robot's $\mathrm{CG}$ :

$$
\dot{\mathbf{Z}}_{\mathrm{des}}=\mathbf{B}^{-1} \omega
$$

where $\omega=\left[\omega_{x}, \omega_{y}, 0\right]^{T}, \dot{\mathbf{Z}}_{\text {des }}$ is a $3 \times 1$ vector of vertical velocity $\left\{\dot{z}_{\operatorname{des}_{j}}\right\}$ of every foot $j$ supporting the robot body, and $\mathbf{B}$ is a $3 \times 3$ matrix if three legs are in support, of the form $\mathbf{B}=\left\{b_{i j}\right\}$ with $b_{1 j}=y_{j}^{-1}, b_{2 j}=-x_{j}^{-1}, b_{3 j}=1$ for every foot $j$ in contact with the ground.

By solving (28) and representing desired CG angular velocities $\omega_{x}$ and $\omega_{y}$ in terms of stability-margin variations, as in (27), the vertical foot speeds of the supporting legs, as desired to compensate for the stability decrease during a leg's transfer phase, are given by

$$
\begin{aligned}
\dot{z}_{\operatorname{des}_{j}}=-\frac{\dot{S}_{\mathrm{NDESM}}}{R}[ & K_{s x}\left(x_{j+1 \bmod 3}^{-1}-x_{j+2 \bmod 3}^{-1}\right) \\
& \left.+K_{s y}\left(y_{j+1 \bmod 3}^{-1}-y_{j+2 \bmod 3}^{-1}\right)\right]
\end{aligned}
$$

where $R=-y_{1}^{-1} x_{3}^{-1}+y_{1}^{-1} x_{2}^{-1}-y_{2}^{-1} x_{1}^{-1}+y_{2}^{-1} x_{3}^{-1}+y_{3}^{-1} x_{1}^{-1}$ $-y_{3}^{-1} x_{2}^{-1}$ and "mod" stands for the function modulus.

Therefore, as modified to control stability, the complete active-compliance equation for each supporting leg is stated as

$\dot{\mathbf{q}}_{c}=\mathbf{J}^{-1}\left[\mathbf{K}_{\mathbf{p}}\left(\mathbf{X}_{\mathrm{des}}-\mathbf{X}\right)+\mathbf{K}_{\mathbf{f}}\left(\mathbf{F}_{\mathrm{des}}-\mathbf{F}\right)+\dot{\mathbf{Z}}_{\mathrm{des}}+\dot{\mathbf{X}}_{\mathrm{des}}\right]$.

The compliant motion achieved through this equation is a trade off, balancing desired gait position, force distribution, and robot stability. The final active-compliance controller is illustrated in Fig. 9.

The performance of the modified active-compliance controller can be analyzed by expressing (30) in Cartesian workspace coordinates and rearranging by assuming that the 


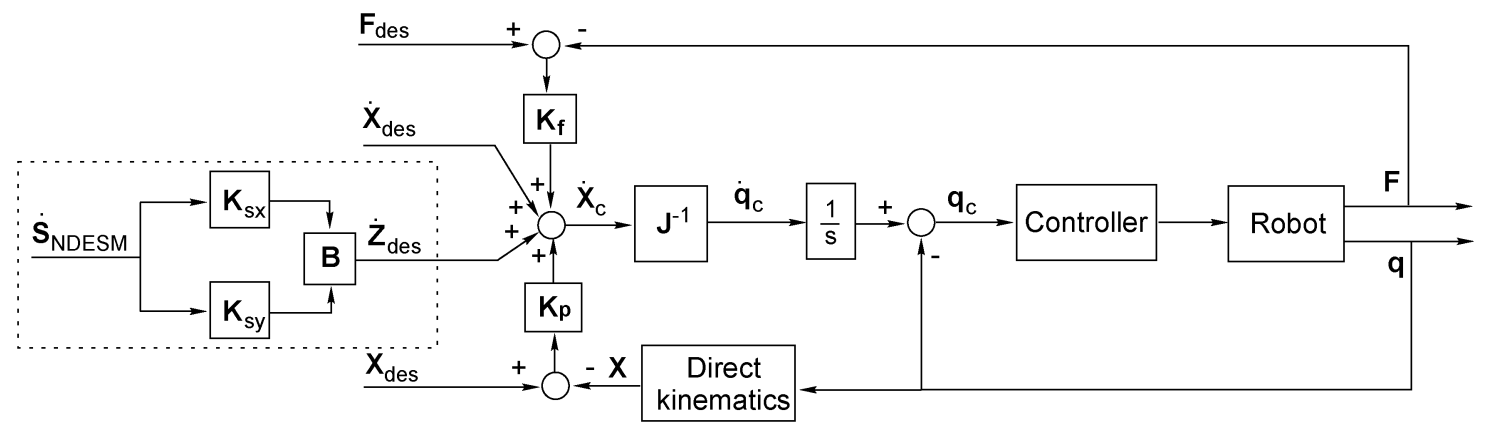

Fig. 9. Block diagram of the active-compliance controller with a stability-dependent term (dashed square).

velocity controller is nearly perfect, and that the actual foot speeds $\dot{\mathbf{X}}$ are very close to the commanded speeds $\dot{\mathbf{X}}_{c}$

$\mathbf{F}_{\mathrm{des}}-\mathbf{F}=-\mathbf{K}_{\mathbf{p}} \mathbf{K}_{\mathbf{f}}^{-1}\left(\mathbf{X}_{\mathrm{des}}-\mathbf{X}\right)-\mathbf{K}_{\mathbf{f}}^{-1}\left(\dot{\mathbf{X}}_{\mathrm{des}}-\dot{\mathbf{X}}\right)-\mathbf{K}_{\mathbf{f}}^{-1} \dot{\mathbf{Z}}_{\mathrm{des}}$.

Equation (31) can be also expressed as

$$
\mathbf{F}_{\text {des }}-\mathbf{F}=-\mathbf{K}_{\mathbf{p}} \mathbf{K}_{\mathbf{f}}^{-1}\left(\mathbf{X}_{\text {des }}-\mathbf{X}\right)-\mathbf{B}(t)\left(\dot{\mathbf{X}}_{\text {des }}-\dot{\mathbf{X}}\right)
$$

where $\mathbf{B}(t)$ is a time-variable damping of the form

$$
\mathbf{B}(t)=\mathbf{K}_{\mathbf{f}}^{-1}\left[\mathbf{I}+\left[K_{s x} \boldsymbol{\alpha}(\mathbf{X})+K_{s y} \boldsymbol{\beta}(\mathbf{X})\right] \dot{S}_{\mathrm{NDESM}}(t) \mathbf{d X}^{-1}\right.
$$

where $\mathbf{d X}$ is a $3 \times 3$ diagonal matrix of elements $\left\{d x_{j j}\right\}=$ $\dot{X}_{\text {des }}-\dot{X}$. Also, $\alpha(\mathbf{X})=\left\{\alpha_{j j}\right\}$ and $\beta(\mathbf{X})=\left\{\beta_{j j}\right\}$ are diagonal matrices with elements

$$
\begin{aligned}
\alpha_{j j} & =\left(x_{j+1 \bmod 3}^{-1}-x_{j+2 \bmod 3}^{-1}\right) / R \\
\beta_{j j} & =\left(y_{j+1 \bmod 3}^{-1}-y_{j+2 \bmod 3}^{-1}\right) / R .
\end{aligned}
$$

Equations (32) and (33) correspond to the equation of motion of a mass-spring-damper system with variable damping. Again, $\mathrm{CR}^{-1}=\mathbf{K}_{\mathbf{p}} \mathbf{K}_{\mathbf{f}}^{-1}$ is the effective stiffness and $\mathbf{B}(t)$ the effective damping of the system, which is a time-varying function of the stability margin. Considering that the term $\dot{S}_{\mathrm{NDESM}}(t)$ can be positive or negative, there exists the possibility that the effective damping coefficient $B(t)$ changes from positive to negative values. In the case that the damping is negative, the damper adds energy to the system, which suggests that the system motion has a divergent tendency. On the other hand, when $B(t)>$ 0 , the damper removes energy from the system, implying that the system motion has a convergent tendency. Therefore, gains $K_{s x}$ and $K_{s y}$ have to be tuned properly to avoid the system response oscillate in a limit cycle. Gain tuning is explained in Section IV-B.

To show how the proposed scheme of active compliance with stability compensation provides better adaptation to both the environment and disturbances, the SILO4 robot was placed on a surface having an inclination of $10^{\circ}$ and commanded to start walking, using first a conventional active-compliance controller ( $K_{p}=1.0 \mathrm{~s}^{-1}, K_{f}=0.4 \mathrm{~mm} / \mathrm{s} / \mathrm{N}$ for every leg), and then the proposed active-compliance controller with stability compensation $\left(K_{p}=0.8 \mathrm{~s}^{-1}, K_{f}=0.4 \mathrm{~mm} / \mathrm{s} / \mathrm{N}, K_{s x}=2.0 \mathrm{~m}^{-1}\right.$, $K_{s y}=6.5 \mathrm{~m}^{-1}$ for every leg). In both cases, at the moment of leg lift, the robot fell slightly downhill because of the perturbing impulse of the leg in transfer. Two consequences are shown in
Fig. 10. When the conventional active-compliance controller is used (thin line), the stability margin decreases, and then it remains at a low level, as shown in Fig. 10(c). However, when the proposed active-compliance controller with stability compensation is used (thick line), the robot recovers from the perturbation, and the stability margin increases after a transient response, eventually stabilizing at a stability level even higher than the initial state. If we compare the measured forces of the foot of leg 4 in support as obtained with the two active-compliance controllers, we can observe in Fig. 10(a) that the proposed herein controller makes the foot forces of the supporting legs fluctuate after a transient of $0.7 \mathrm{~s}$ to compensate for the stability losses. This can be also observed in Fig. 10(b), where foot $z$-positions are shown to fluctuate due to foot-speed changes for stability compensation.

\section{EXPERIMENTAL SETUP}

The SILO4 quadruped robot shown in Fig. 1 has been used for experiments to show the improvement in walking afforded by the approach proposed herein. Main features of this robot can be found in [14] and [15].

\section{A. Force Measurement and Processing}

In order to carry out the experiments that are reported in Section V, the reaction forces at the feet have been measured and processed to accomplish three main issues:

force feedback for active-compliance control;

computation of $S_{\mathrm{NDESM}}$ and its time derivative;

determination of ground contact.

Three-axial force sensors based on quartz transducers are located at each foot to measure the ground-reaction forces, which however have been filtered differently as it was required for each task.

1) Force feedback for active-compliance control: Active compliance with stability-compensation control is performed during the cycle period when three legs are on the ground. Foot force-feedback is required, as (30) shows. The raw force is filtered by means of a first-order low-pass filter (LPF) (time constant $\tau=0.68 \mathrm{~s}$, cutoff frequency $f_{c}=$ $0.49 \mathrm{~Hz}$ ) which achieves a tradeoff between speed of response and smoothness to accomplish a stable active compliance control with a period of $0.01 \mathrm{~s}$. Fig. 11 allows for comparison between raw force from the sensor (thin line) and filtered force (thick line) when the leg is in support phase. 


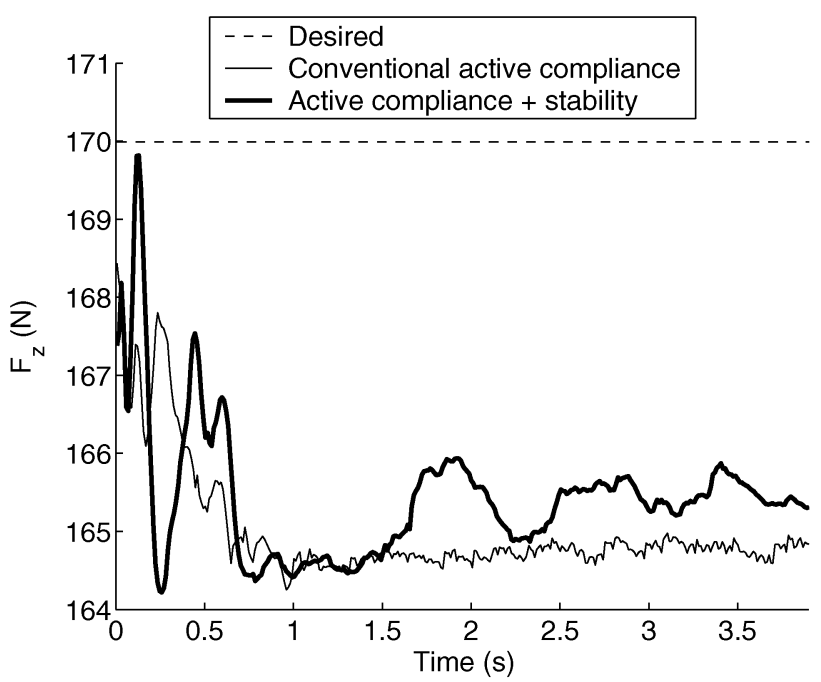

(a)

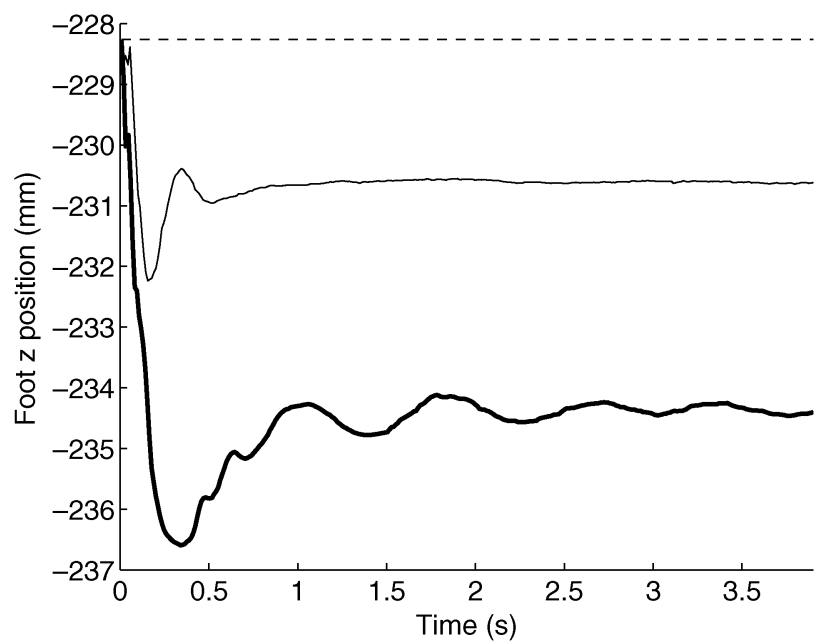

(b)

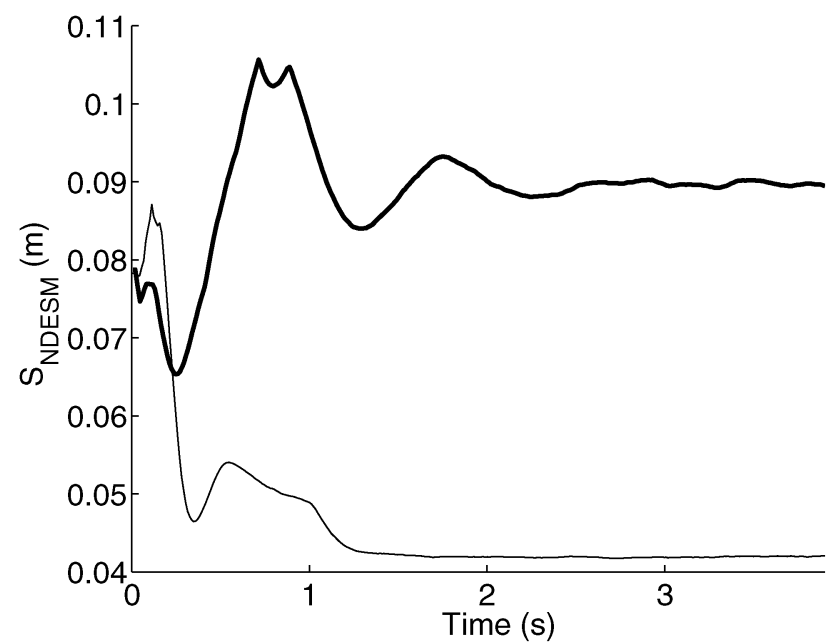

(c)

Fig. 10. Result of applying a conventional active-compliance controller (thin line) versus the proposed combined active-compliance and stability controller (thick line). (a) Measured vertical force at foot 4. (b) $Z$ component of foot 4. (c) Gait stability margin.

2) Computation of $S_{\mathrm{NDESM}}$ and $\dot{S}_{\mathrm{NDESM}}$ : The same filtered foot-force data used for active compliance control is also

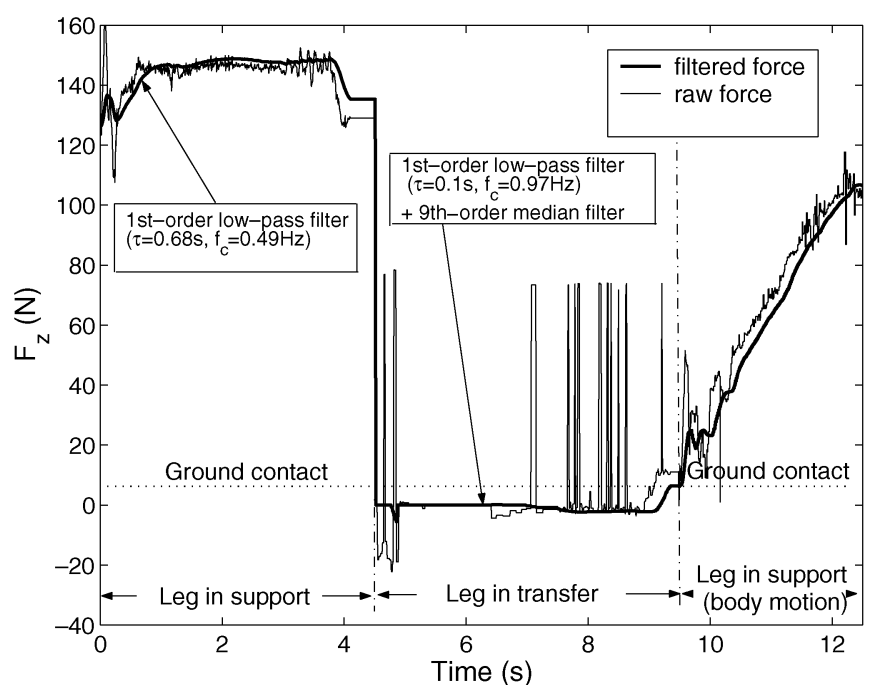

Fig. 11. Measurement of vertical foot force in the leg 1 of the SILO4: raw force (thin line) and filtered force (thick line).

used to compute $S_{\mathrm{NDESM}}$. For $S_{\mathrm{NDESM}}$ computation purposes, only the force of the feet on the ground are considered. The active compliance with stability-compensation controller uses the time derivative of $S_{\text {NDESM }}$ as (27) shows to compensate for stability loses during the time period when three legs are on the ground. To compute $\dot{S}_{\text {NDESM }}$ the value of $S_{\mathrm{NDESM}}$ is filtered by means of a first-order LPF ( $\tau=$ $0.95 \mathrm{~s}, f_{c}=0.2 \mathrm{~Hz}$ ). The time derivative of the resulting $S_{\text {NDESM }}$ is again filtered, using a first-order LPF ( $\tau=$ $\left.0.05 \mathrm{~s}, f_{c}=2.1 \mathrm{~Hz}\right)$. The final $\dot{S}_{\mathrm{NDESM}}$ that has been used for the experiments is a smooth signal that only reflects the significant changes in the stability margin. This allows for stable control.

3) Determination of ground contact: The instant of ground contact at the end of the transfer phase of a leg is determined by foot-force measurement. The noise in the force measurement is reduced by using a first-order LPF ( $\tau=$ $0.1 \mathrm{~s}, f_{c}=0.97 \mathrm{~Hz}$ ). There is also a need to eliminate some different noise that comes from the piezoelectric nature of the force sensors. The crystals of quartz transducers produce an electrical output only when they experience a change in load. For this reason, they cannot perform true static measurements. When the leg is on the air, static force measurement is required, and the sensor sometimes provides false measurements that lead to the determination of a false ground contact. The problem is not solved by the above LPF, nor it can be easily solved by resetting each time the foot is known to be off the ground, because the ground contact instant is detected by the same measurement. We have solved it by using a nine-order median filter before the above LPF. The resulting force measurement is suitable for ground-detection purposes. Fig. 11 allows for comparison between raw force from the sensor (thin line) and filtered force (thick line) during different leg phases. Observe the different noise that takes place during leg transference, which makes it difficult to estimate the instant of ground contact, and how the median filter solves it. Ground contact is found for a reaction force higher than $6 \mathrm{~N}$. 


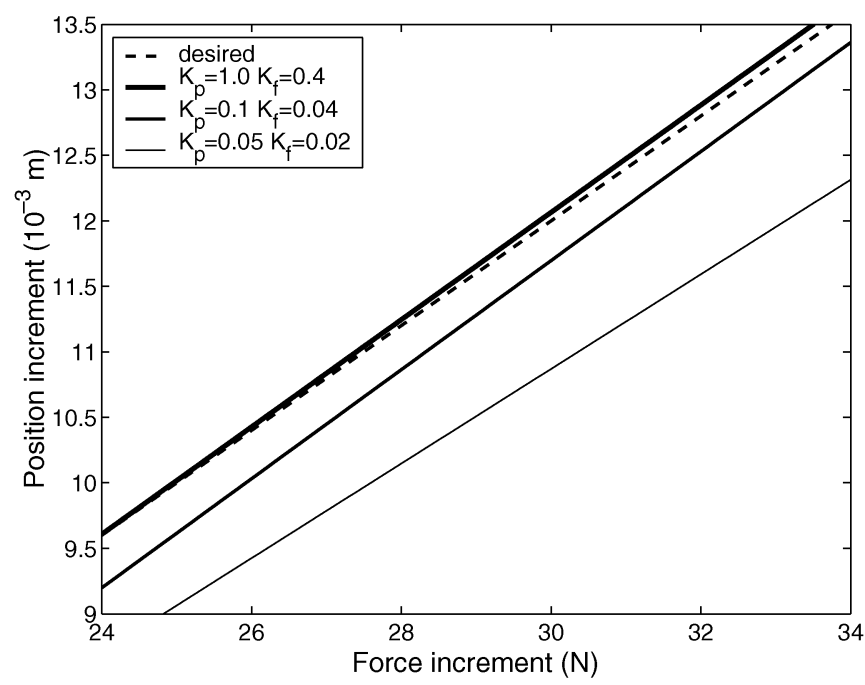

Fig. 12. Effect of different gains $K_{p} K_{f}$ for a CR of $0.4 \mathrm{~mm} / \mathrm{N}$.

\section{B. Tuning of Gains}

As explained in Section III-B, the proposed active compliance with stability-compensation controller can be modeled as a mass-spring-damper system with a time-varying damping coefficient as in (32) which has to comply with an environment that we model by a stiffness $K_{E}$. The effective compliance of the system is $\mathrm{CR}=K_{f} / K_{p}$, and the variable damping of the system is $B(t)$, described in (33).

It is necessary to adequately choose $\mathbf{K}_{\mathbf{p}}$ and $\mathbf{K}_{\mathbf{f}}$ in respect of the characteristics of the environment. The steady-state value of the foot position is

$$
X_{\infty}=\frac{X_{\mathrm{des}}+\frac{K_{f} K_{E}}{K_{p}} X_{E}}{1-\frac{K_{f} K_{E}}{K_{p}}} .
$$

Since the environmental stiffness $K_{E}$ is typically high, $X_{\infty} \approx$ $X_{E}$, and therefore, we should tune these gains to have large $K_{f}$ and small $K_{p}$, so that $K_{E} \gg K_{p} / K_{f}$. However, there is an upper limit in $K_{f}=K_{f, \max }$ above which the system gets unstable. In our system, $K_{f, \max }=0.6 \mathrm{~mm} / \mathrm{s} / \mathrm{N}$. Therefore, for this application, a $K_{f}$ as close as possible to the limit $K_{f, \max }$ should be used. Considering that the feet on the ground should have to correct their position to comply with the environment, and considering that the feet should correct as a mean $8 \mathrm{~mm}$ to comply with a $20 \mathrm{~N}$ force increment, then the desired CR of our system is $0.4 \mathrm{~mm} / \mathrm{N}$. Fig. 12 shows the effect of different gains for a $\mathrm{CR}$ of $0.4 \mathrm{~mm} / \mathrm{N}$. One can observe how the system effective compliance gets closer to the desired effective compliance as $K_{f}$ and $K_{p}$ increase. However, the system gets unstable for $K_{f}=0.6 \mathrm{~mm} / \mathrm{s} / \mathrm{N}$, and therefore, we choose for the experiments $K_{f}=0.4 \mathrm{~mm} / \mathrm{s} / \mathrm{N} 0.4 \mathrm{~mm} / \mathrm{s} / \mathrm{N}$ and $K_{p}=1.0 \mathrm{~s}^{-1}$.

In the transient response of the system, the time-varying damping coefficient can lead to a limit cycle. Therefore, gains $K_{s x}$ and $K_{s y}$ have to be tuned to ensure that $B(t)$ is always positive. This condition leads to the following equation:

$$
K_{s x} \boldsymbol{\alpha}(\mathbf{X})+K_{s y} \boldsymbol{\beta}(\mathbf{X}) \geq-\max \left(\frac{1}{\dot{S}_{\mathrm{NDESM}}(t)} \mathbf{d X}\right) .
$$

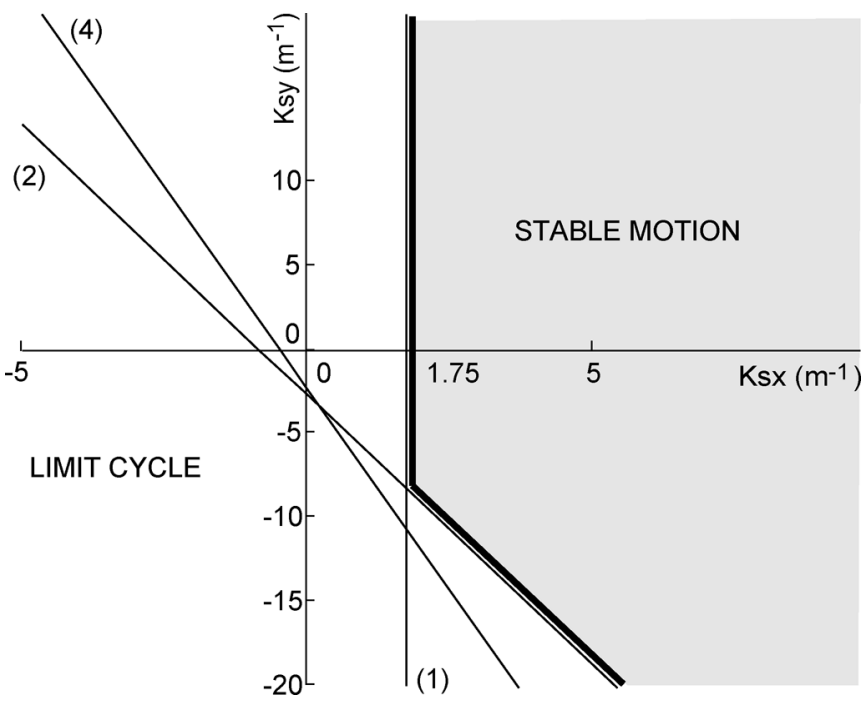

Fig. 13. Areas of stable motion and limit cycle as a function of gains $K_{s x}$ and $K_{s y}$.

\section{TABLE I}

LOOKUP TABLE FOR GAIT OPTIMIZATION WHEN EXTERNAL FORCES EXIST

\begin{tabular}{|c|c|c|c|c|c|c|c|}
\hline $\begin{array}{c}F_{x} \\
(\mathrm{~N})\end{array}$ & $\begin{array}{c}\mathrm{d} \\
(\mathrm{m})\end{array}$ & $\begin{array}{c}\mathrm{x} 1 \\
(\mathrm{~m})\end{array}$ & $\begin{array}{c}\mathrm{x} 2 \\
(\mathrm{~m})\end{array}$ & $\begin{array}{c}\mathrm{x} 3 \\
(\mathrm{~m})\end{array}$ & $\begin{array}{c}\mathrm{x} 4 \\
(\mathrm{~m})\end{array}$ & $\begin{array}{c}\mathrm{h} \\
(\mathrm{m})\end{array}$ & $\begin{array}{c}S_{\text {gait }} \\
(\mathrm{m})\end{array}$ \\
\hline \hline 0 & 0.2 & 0.250 & 0.450 & -0.250 & -0.050 & 0.228 & 0.018 \\
-25 & 0.192 & 0.226 & 0.418 & -0.258 & -0.065 & 0.209 & 0.018 \\
-50 & 0.180 & 0.192 & 0.372 & -0.269 & -0.088 & 0.213 & 0.018 \\
-75 & 0.171 & 0.164 & 0.336 & -0.278 & -0.107 & 0.208 & 0.018 \\
-100 & 0.161 & 0.133 & 0.294 & -0.289 & -0.127 & 0.208 & 0.018 \\
-200 & 0.100 & 0.050 & 0.150 & -0.350 & -0.250 & 0.179 & 0.018 \\
-250 & 0.047 & 0.024 & 0.071 & -0.402 & -0.355 & 0.061 & 0.018 \\
\hline
\end{tabular}

Fig. 13 shows the area of acceptable values of $K_{s x}$ and $K_{s y}$ that ensure the stable motion of the system. Thin lines are obtained by applying (37) to legs 1,2 , and 4, respectively (designed by numbers inside brackets), while leg 3 is in transfer. Similar lines are obtained for the other legs in transfer phase. The thick line represents the limit curve for stable motion of the walking robot as a function of gains $K_{s x}$ and $K_{s y}$. Our system has been tuned with gains $K_{s x}=2.0 \mathrm{~m}^{-1}$ and $K_{s y}=6.5 \mathrm{~m}^{-1}$.

\section{EXPERIMENTS WITH THE SILO4 QUADRUPED ROBOT}

Three experiments have been carried out using the SILO4 quadruped robot to show the improvement achieved in walking performance.

The first experiment is designed to focus upon the adaptation to external or environmental forces. The SILO4 robot walks on natural ground, and it is affected by an external force, achieved by attaching the robot to a load (see Fig. 1). $S_{\text {NDESM }}$ is computed while the robot walks against the disturbance using a two-phase discontinuous gait. The robot finally tumbles down due to a 50-N force. A digital video of this experiment is available at $\mathrm{http}: / /$ ieeexplore.ieee.org (see Video 1). Then, the experiment is repeated using the gait-adaptation approach, which modifies the gait pattern for different external forces, as listed in Table I, thus reducing CG height and leg stroke to maximize the stability margin. In this experiment, the robot pulls the same 


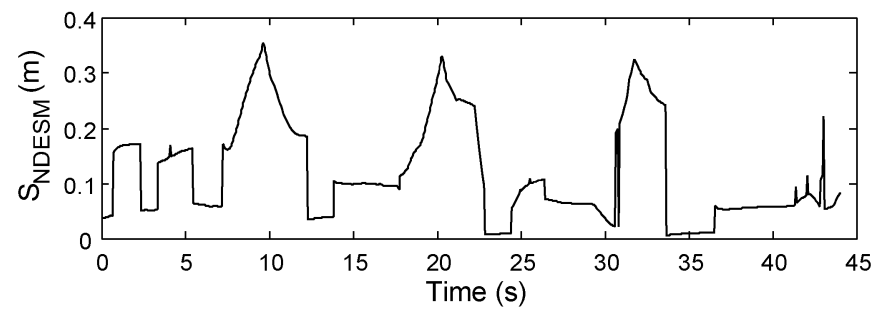

(a)

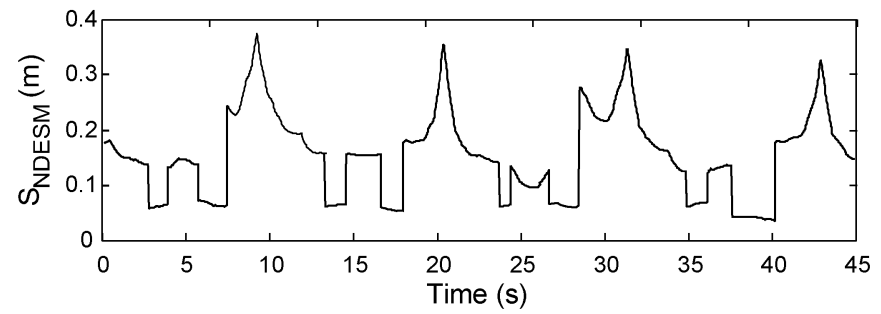

(b)

Fig. 14. Time evolution of $S_{\mathrm{NDESM}}$ for the SILO4 robot walking against a 50-N disturbance on natural ground. (a) Standard gait. (b) Adapted gait using the proposed approach.

load that made the robot tumble down in the previous experiment at a force of $50 \mathrm{~N}$. See Video 2 at http://ieeexplore.ieee.org.

Fig. 14(a) shows the evolution of the stability margin during the experiment in Video 1, when the SILO4 robot walks against the disturbance using a standard two-phase discontinuous gait. The asymmetry of the stability margin increases with time, the stability margin dropping ever farther during the transfer phase of the rear legs (because the destabilizing effects of leg transfer and force disturbance combine). Let us compare Fig. 14(a) with Fig. 14(b) which shows the evolution of the stability margin during the experiment in Video 2, when the SILO4 robot walks using the proposed adaptive gait. As a result of adapting the gait to a $50-\mathrm{N}$ force, the stability margin is symmetrical for the transfer phases of both the rear and the front legs. As a result of the adaptation to a $50-\mathrm{N}$ force, the gait is more stable than in Fig. 14(a), increasing the gait stability margin by $70 \%$ in this experiment. As shown in Table I, the use of the proposed gaitparameter-adaptation approach increases the robot's robustness to external disturbances from a 50-N load up to a $200-\mathrm{N}$ load, that is, four times the perturbation in this particular robot. Note that this robot is not able to pull a load higher than $200 \mathrm{~N}$ because the CG height required for such forces is not admissible due to kinematics.

The second experiment is aimed at showing how body regulation improves robot stability throughout the gait cycle, by tracking the stability margin as the SILO4 robot walks. Fig. 15 shows three plots representing the stability margin for three cases:

Case 1) the robot walks without active compliance, depicted in a solid thin line;

Case 2) the robot gait is controlled under a conventional active-compliance controller $\left(K_{p}=1.0 \mathrm{~s}^{-1}, K_{f}=\right.$ $0.4 \mathrm{~mm} / \mathrm{s} / \mathrm{N}$ for every leg in support), plotted in a dashed thin line;

Case 3) The robot gait is controlled by the proposed active-compliance controller with stability compensation $\left(K_{p}=1.0 \mathrm{~s}^{-1}, K_{f}=0.4 \mathrm{~mm} / \mathrm{s} / \mathrm{N}\right.$,

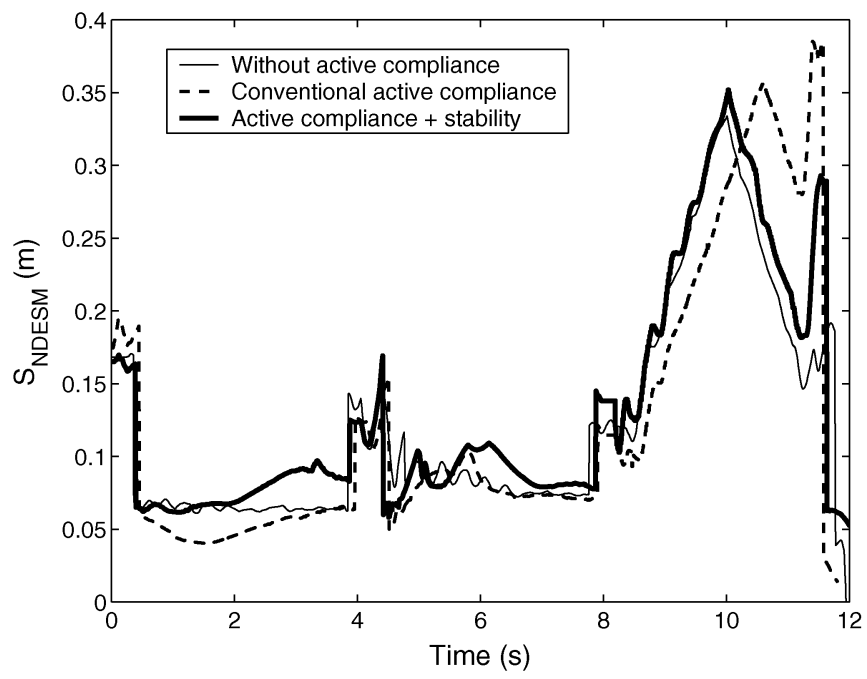

Fig. 15. Stability margin along half a gait cycle for three cases: without active compliance (solid thin line); with conventional active compliance (dashed thin line); and with active compliance and stability compensation (solid thick line).

$$
K_{s x}=2.0 \mathrm{~m}^{-1}, K_{s y}=6.5 \mathrm{~m}^{-1} \text { for every leg in }
$$
support). This last case is drawn in a thick line.

As the figure shows, the stability margin fluctuates as the robot walks without any kind of active compliance, because of the rigidity of the machine. The application of an active-compliance controller clearly smoothes the walk, as depicted in a dashed line. However, the compliant motion causes the stability margin to decrease at the beginning of the leg transfer. The application of the active-compliance controller with stability compensation improves the resulting motion, achieving a better gait stability margin while maintaining compliant motion. Note that Fig. 15 only shows the improvement in gait stability margin when the proposed active-compliance with stability compensation is used (not the complete dual approach). As it was explained in Section III (see Fig. 4), the goal of the body regulation by active compliance with stability compensation is to compensate for stability-margin loses. It is not assumed to increase the overall stability margin above the conventional method, but to avoid the stability margin to decrease, maintaining it leveled. If we compare the gait stability margin for the three cases in Fig. 15, that is, the minimum stability margin that takes place at $t=1.8 \mathrm{~s}$ during the rear leg's transfer phase, we can see that the gait stability margin when the conventional active compliance is used is $35 \mathrm{~mm}$, and it is increased by $25 \mathrm{~mm}$ more when using the proposed approach. That represents an improvement of $70 \%$ in gait stability margin. But how is the improvement in gait stability margin related to the improvement in walking performance? As Fig. 15 shows, even the conventional activecompliance controller keeps enough stability margin. However, if during this period of leg transference a perturbation is produced or robot speed increases, the controller that achieves the poorer gait stability margin is more likely to allow the robot to tumble down precisely during this period of time. Therefore, the improvement in gait stability margin is a measurement of the robustness to unexpected dynamic effects. See Videos 3 and 4, showing the improvement in gait stability of the proposed approach, at http://ieeexplore.ieee.org. Video 3 shows how the 


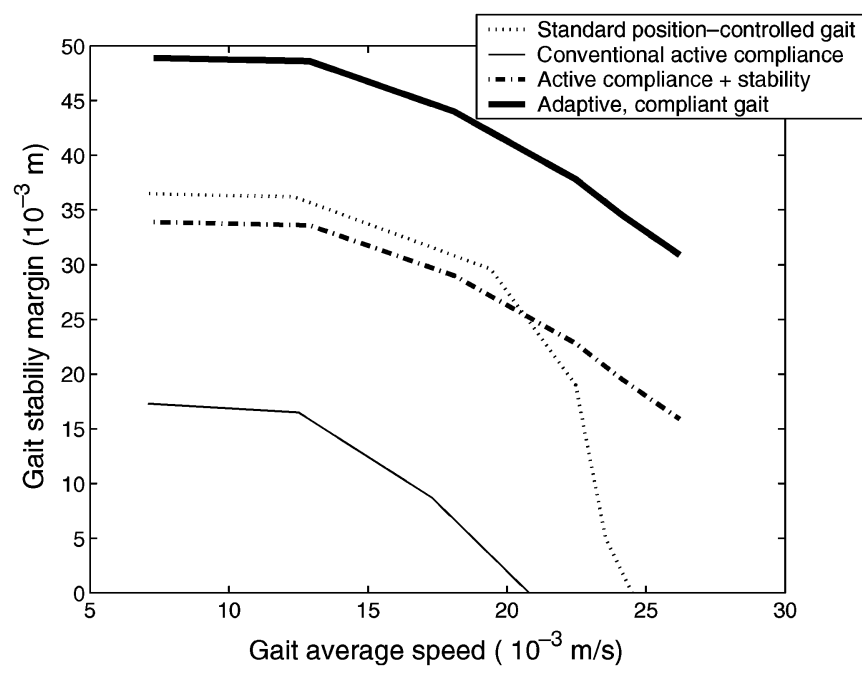

Fig. 16. Curves of gait stability versus average gait speed for a ground inclined at $5^{\circ}$ for cases (a) standard position-controlled gait (dotted line), (b) with conventional active compliance (solid thin line), (c) with active compliance and stability compensation (dash-dot thick line), and (d) the complete adaptive compliant gait (thick line).

robot gets unstable when it walks uphill using a conventional active-compliance controller. Then, the motion is repeated in Video 4 using the proposed active-compliance controller with stability compensation, resulting in a stable and compliant motion.

The third experiment is aimed to answer the above question of what happens during the period of minimum stability margin if robot speed increases. This experiment shows the improvement in gait speed that can be achieved by using the proposed approach. In this experiment, the robot walks uphill on a $5^{\circ}$ slope. The gait stability margin has been plotted for different average gait speeds in Fig. 16. As a result, four curves have been obtained, which show the evolution of the gait stability margin as gait speed increases for a standard position-controlled gait (dotted line), an active-compliance-controlled gait (thin solid line), an active-compliance-controlled gait with stability compensation (thick dash-dotted line), and the complete adaptive gait with body regulation using active compliance with stability compensation (thick solid line). This figure shows how the gait stability margin decreases as speed increases, just because leg acceleration increases, leg inertia increases, and this lowers the dynamic stability margin, which is a measurement of how much dynamic perturbation the leg applies on the robot CG during leg transference. As this figure shows, the conventional active-compliance controller does not do a good job for inclined ground, because the compliant motion reduces gait stability, and the gait becomes unstable for an average gait speed of about $21 \mathrm{~mm} / \mathrm{s}$. The position-controlled gait is more stable than the active-compliance-controlled gait with stability compensation at low speed, due to the slow transient response of the proposed controller; at high speed, however, the rigidity of the position-controlled gait produces wide oscillations that make the gait unstable. The proposed active compliance with stability compensation, on the other hand, achieves stable motion up to the highest average speed that the actuators allow. Fig. 17 compares the stability margin along a gait cycle for the robot walking at $24 \mathrm{~mm} / \mathrm{s}$ using a position-based controller (dashed

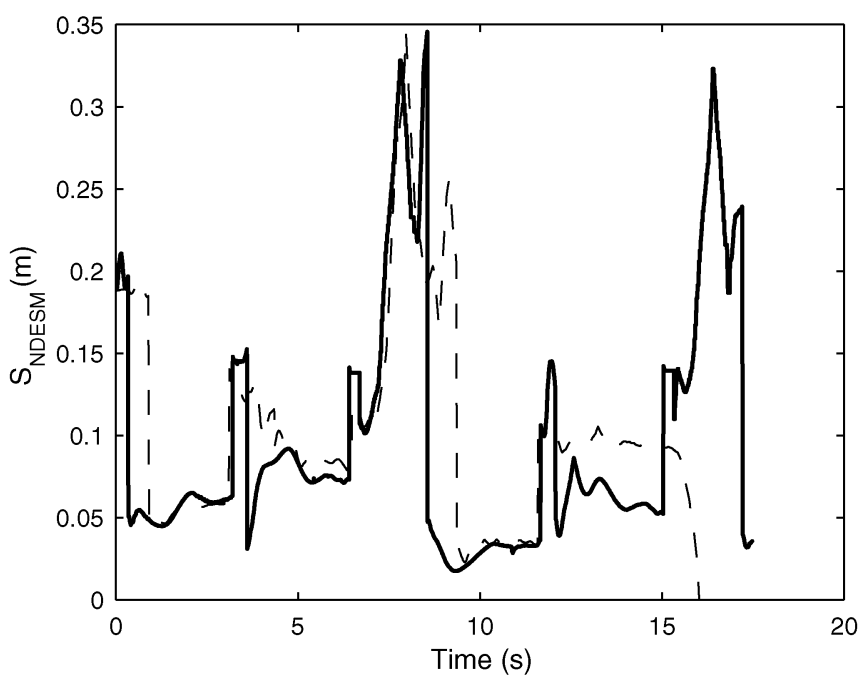

Fig. 17. Stability margin along a gait cycle walking at $24 \mathrm{~mm} / \mathrm{s}$ on a terrain inclined at $5^{\circ}$ : without active compliance (dashed line) and with active compliance and stability compensation (solid line).

line), and using the proposed active compliance controller with stability compensation (solid line). As this figure shows, the stability margin at this speed for a position-controlled gait fluctuates and reaches zero just before the second body-support phase. However, using the approach proposed herein, the gait remains compliant and stable. Finally, the thick solid line in Fig. 16 represents the curve of the gait stability margin for different average gait speeds when the complete dual approach proposed herein is used to control the robot's motion. The gait-parameter adaptation added to the active-compliance controller with stability compensation improves gait stability for every average gait speed by $15 \%$ for the worst case (low speed). The gait stability margin is much larger than for other methods for the same speeds, therefore the robot is more stable and more capable of supporting unexpected perturbations. Also, as speed increases, for speeds that other methods became unstable, this method keeps at values comparable with the best stability values of other methods. This experiment has been done for increasing gait speeds until the actuator speed limits were found, at $26.2 \mathrm{~mm} / \mathrm{s}$. Even when the actuator limits are found and we simply cannot go on with the experiments, if one imagines the tendency of the curve, one can see that it could keep stable up to gait speeds of $40-50 \mathrm{~mm} / \mathrm{s}$, which doubles the maximum speed of conventional approaches.

Considering that the main drawback of walking machines compared with wheeled and tracked machines is their low speed and poor stability, the results presented here show the gait-adaptation method is a relevant improvement in the control of walking robots and their adaptation to natural environments. Video 5 showing the SILO4 walking uphill in natural ground by using the complete gait adaptation approach (gait parameter adaptation and active compliance with stability compensation) is available at http://ieeexplore.ieee.org.

\section{CONCLUSIONS}

A method for achieving a compliant adaptive gait for a quadruped has been proposed in this paper. The proposed 
approach is divided into two steps. The first step finds the gait parameters that maximize robot stability in the presence of environmental perturbations, like external forces or sloping terrain. The gait-parameter-adaptation method is inspired by the natural gait adaptation carried out by humans and animals to balance external forces or the effect of sloping terrain. The second step of the proposed approach is a body-regulation system to counteract internal leg dynamics and impulsive perturbations during leg-transfer phases that usually reduce the gait stability margin, thus increasing the probability of tumbling down. The body-regulation system is based on the new active-compliance controller herein proposed that improves conventional active compliance by means of a stability-compensation term. This compensation term is based on a dynamic energy-stability measurement that considers the impact energy a body can withstand.

Experiments using the SILO4 robot have been performed indoors and outdoors to show the improvement of the proposed approach. The proposed gait-parameter adaptation compensates for destabilizing effects, increasing the SILO4 robot's robustness to external disturbances to $70 \%$. The proposed active-compliance controller with stability compensation also counteracts impulsive perturbations that, augmented by the compliant behavior of conventional active-compliance controllers cause instability, especially when the robot walks on inclined surfaces. The proposed approach also allows average gait speed to be increased up to actuator limits, improving on position-based gait controllers by about $15 \%$ for the worst case. The active-compliance controller with stability compensation does not outperform position-based controllers at low speeds due to the slow transient response of the compliant controller. However, the coalition of the gait-parameter-adaptation approach and the activecompliance controller with stability compensation, presented in this paper, do improve on position-based controllers, even at low speed, by about $32 \%$. The proposed active compliance with stability-compensation controller improves on conventional active-compliance controllers by about $102 \%$ on inclined ground.

\section{APPENDIX}

\section{COMPUTATION OF $S_{\mathrm{NDESM}}$}

Let us consider a walking robot during its motion. At a given instant, an external impact causes the robot to tumble around one edge of its support polygon. Fig. 2 depicts the CG of a robot during the tumble around the edge of its support polygon, given by the line connecting footprints $i$ and $i+1$. This edge is inclined at an angle $\psi$ from the horizontal plane due to terrain inclination. If the moment around this rotational axis caused by the resultant forces and moments of robot/ground interaction, $\mathbf{F}_{\mathbf{R}}$ and $\mathbf{M}_{\mathbf{R}}$, is able to compensate for the destabilizing effect, the robot could maintain stability. If, on the contrary, the effect cannot be compensated for, the robot will lose stability. Therefore, the instant of critical stability occurs when the moment of robot/ground interaction forces and moments around the rotation axis vanishes. At that time, the $\mathrm{CG}$ is located inside a critical plane that forms an angle $\phi$ with the vertical plane (position (2) in Fig. 2).

At the initial position (1) before the tumble, the CG is subject to inertial forces and moments $\left(\mathbf{F}_{\mathbf{I}}\right.$ and $\left.\mathbf{M}_{\mathbf{I}}\right)$, gravitational forces and moments $\left(\mathbf{F}_{\mathbf{G}}\right.$ and $\left.\mathbf{M}_{\mathbf{G}}\right)$, and manipulation forces and moments $\left(\mathbf{F}_{\mathbf{M}}\right.$ and $\left.\mathbf{M}_{\mathbf{M}}\right)$. The perturbing effects of a leg in transfer phase can be also considered as manipulation terms. Assuming that the dynamics of the legs in the support phase is negligible relative to the body dynamics, the resultant force and moment of robot/ground interaction are given by

$$
\begin{aligned}
\mathbf{F}_{\mathbf{R}} & =\mathbf{F}_{\mathbf{G}}+\mathbf{F}_{\mathbf{M}}-\mathbf{F}_{\mathbf{I}} \\
\mathbf{M}_{\mathbf{R}} & =\mathbf{M}_{\mathbf{G}}+\mathbf{M}_{\mathbf{M}}-\mathbf{M}_{\mathbf{I}} .
\end{aligned}
$$

The mechanical energy increase experienced by the CG during the tumble from position (1) to position (2) is given by the following energy balance:

$$
E_{i}=V_{2}-V_{1}+K_{2}-K_{1}
$$

where $V_{1}$ and $K_{1}$ are the potential and kinetic energies of the CG, respectively, before the tumble (1), and $V_{2}$ and $K_{2}$ are the potential and kinetic energy of the CG at the critical plane. Inside the critical plane, the rotational speed of the $\mathrm{CG}$ is zero at this time, therefore

$$
E_{i}=V_{2}-V_{1}-K_{1}
$$

The increase of potential energy, $V_{2}-V_{1}$, is the sum of potential energy due to gravity, $\mathbf{F}_{\mathbf{G}}$, and the rest of forces and moments, $\mathbf{F}_{\mathbf{R I}}$ and $\mathbf{M}_{\mathbf{R}}$, that is

$$
\begin{aligned}
V_{2}-V_{1} & =\Delta V_{G}+\Delta V_{F}+\Delta V_{M} \\
\Delta V_{G} & =m g h \\
\Delta V_{F} & =\int_{\theta_{1}}^{\theta_{2}}\left(\mathbf{F}_{\mathbf{R I}} \times \mathbf{R}\right) \cdot \mathbf{e}_{\mathbf{i}} d \theta
\end{aligned}
$$

where

$$
\mathbf{F}_{\mathbf{R I}}=\mathbf{F}_{\mathbf{R}}-\mathbf{F}_{\mathbf{G}}
$$

is the nongravitational component of $\mathbf{F}_{\mathbf{R}}$. Finally

$$
\Delta V_{M}=\int_{\theta_{1}}^{\theta_{2}}\left(\mathbf{M}_{\mathbf{R}} \cdot \mathbf{e}_{\mathbf{i}}\right) d \theta .
$$

To compute the kinetic energy of the system before the tumble, the following equation must be solved:

$$
K_{1}=\frac{1}{2} I_{i} \omega_{i}^{2}
$$

where $I_{i}$ is the moment of inertia around the rotation axis, which is known, and $\omega_{i}$ is the angular speed of the robot before the tumble, which is obtained from

$$
\omega_{i}=\frac{L_{i}}{I_{i}} .
$$


Let us consider the speed of the CG before the tumble (1), $\mathbf{v}_{\mathrm{CG}}$. Then, the angular momentum $L_{i}$ is computed from

$$
L_{i}=\left(\mathbf{R} \times m \mathbf{v}_{\mathrm{CG}}\right) \cdot \mathbf{e}_{i}
$$

where $m$ is the total mass of the robot and its manipulator system. Then the kinetic energy of the system before the tumble can be obtained by substituting (48) and (49) in (47).

Thus, the term $E_{i}$ is obtained by substituting (42)-(47) into (40)

$$
\begin{aligned}
E_{i}=m g|\mathbf{R}|(\cos \phi & -\cos \varphi) \cos \Psi \\
& +\left(\mathbf{F}_{\mathbf{I}} \cdot \mathbf{t}\right)|\mathbf{R}| \theta+\left(\mathbf{M}_{\mathbf{I}} \cdot \mathbf{e}_{\mathbf{i}}\right) \theta-\frac{1}{2} I_{i} \omega_{i}^{2} .
\end{aligned}
$$

$E_{i}$ is the stability level, and physically means the increase of mechanical energy of the CG when pivoting around the edge $i$ of the support polygon.

The final expression of $S_{\mathrm{NDESM}}$ is

$$
S_{\mathrm{NDESM}}=\frac{\min \left(E_{i}\right)}{m g}
$$

where $E_{i}$ is the stability level, given by (50).

\section{REFERENCES}

[1] P. Gonzalez de Santos and M. Jimenez, "Generation of discontinuous gaits for quadruped walking machines," J. Robot. Syst., vol. 12, no. 9, pp. 599-611, 1995.

[2] D. Wettergreen and C. Thorpe, "Developing planning and reactive control for a hexapod robot," in Proc. IEEE Int. Conf. Robot. Autom., 1996, pp. 2718-2723.

[3] J. Estremera and P. Gonzalez de Santos, "Free gaits for quadruped robots over irregular terrain," Int. J. Robot. Res., vol. 21, no. 2, pp. 115-130, 2002.

[4] K. Tsujita, K. Tsuchiya, and Onat, "Adaptive gait pattern control of a quadruped locomotion robot," in Proc. IEEE/RSJ Int. Conf. Intell. Robots Syst., 2001, pp. 2318-2325.

[5] M. Lewis and Bekey, "Gait adaptation in a quadruped robot," Auton. Robots, vol. 12, no. 3, pp. 301-312, 2002.

[6] Y. Fukuoka, H. Kimura, and A. Cohen, "Adaptive dynamic walking of a quadruped robot on irregular terrain based on biological concepts," Int. J. Robot. Res., vol. 22, no. 3-4, pp. 187-202, 2003.

[7] H. Kimura and Y. Fukuoka, "Biologically inspired adaptive dynamic walking in outdoor environment using a self-contained quadruped robot: 'Tekken2',' in Proc. IEEE/RSJ Int. Conf. Intell. Robots Syst., 2004, pp. 986-991.

[8] A. Kuo, "The relative roles of feedforward and feedback in the control of rhythmic movements," Motor Control, no. 6, pp. 129-145, 2002.

[9] D. Gorinevsky and A. Schneider, "Force control in locomotion of legged vehicles over rigid and soft surfaces," Int. J. Robot. Res., vol. 9, no. 2, pp. 4-23, 1990.

[10] D. Bevly, S. Dubowsky, and C. Mavroidis, "A simplified Cartesiancomputed torque controller for highly geared systems and its application to an experimental climbing robot," J. Dyn. Syst., Meas., Control, vol. 122 , pp. 27-32, 2000.

[11] F. Palis, V. Rusin, and A. Schneider, "Adaptive impedance/force control of legged robot systems," in Proc. Int. Conf. Climbing, Walking Robots, Karlsruhe, Germany, 2001, pp. 323-329.
[12] E. Garcia and P. Gonzalez de Santos, "An improved energy stability margin for walking machines subject to dynamic effects," Robotica, vol. 23, no. 1, pp. 13-20, 2005.

[13] E. Garcia, J. Estremera, and P. Gonzalez de Santos, "A comparative study of stability margins for walking machines," Robotica, vol. 20, pp. 595-606, 2002.

[14] P. Gonzalez de Santos, J. Galvez, J. Estremera, and E. Garcia, "SILO4-A true walking robot for the comparative study of walking machine techniques," IEEE Robot. Autom. Mag., vol. 10, no. 4, pp. 23-32, 2003

[15] “The SILO4 Walking Robot,” Ind. Autom. Inst., C.S.I.C., 2002 [Online]. Available: http://www.iai-csic.es/users/silo4/

[16] H. Tsukagoshi and S. Hirose, "Intermittent crawl gait for quadruped walking vehicles on rough terrain," in Proc. Int. Conf. Climbing, Walking Robots, Brussels, Belgium, Nov. 1998, pp. 323-328.

[17] M. Hardt and O. Von Stryk, "Increasing stability in dynamic gaits using numerical optimization," in Proc. 15th IFAC World Congr. Autom. Control, Barcelona, Spain, 2002, pp. 1636-1641.

[18] J. Weingarten, G. Lopes, M. Buehler, R. Groff, and D. Koditschek, "Automated gait adaptation for legged robots," in Proc. IEEE Int. Conf. Robot. Autom., New Orleans, LA, Apr. 2004, pp. 2153-2158.

[19] V. Chankong and Y. V. Haimes, Multiobjective Decision Making Theory and Methodology. New York: Elsevier, 1983.

[20] W. Jiang, A. Liu, and D. Howard, "Optimization of legged robot locomotion by control of foot-force distribution," Trans. Inst. Meas. Control, vol. 26, no. 4, pp. 311-323, 2004.

[21] K. Waldron, "Force and motion management in legged locomotion," IEEE J. Robot. Autom., vol. RA-2, no. 4, pp. 214-220, Aug. 1986.

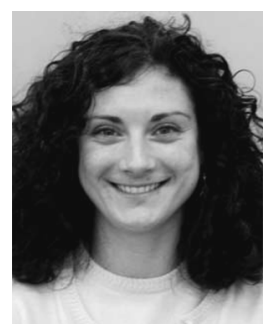

Elena Garcia received the B.E. and Ph.D. degrees from the Polytechnic University of Madrid, Madrid, Spain, in 1996 and 2002, respectively.

She has been a Visiting Scholar at the MIT Leg Laboratory, Cambridge, MA, in 1998, and at the Laboratoire d'Automatique de Grenoble, Grenoble, France, in 2001. She has been a Researcher at the Industrial Automatic Institute-CSIC, Madrid, Spain, since 1997. Her research interests include dynamic stability issues in statically stable gaits of walking robots, dynamic modeling of walking robots, friction in high-geared robotic systems, active compliance, and online generation of high-speed foot trajectories. She has participated in various research projects, such as ROWER, a walking platform for ship building, SILO4, a four-legged locomotion system used as a testbed in most of her work, and DYLEMA, a project focused on a six-legged walking platform for landmine detection and location.

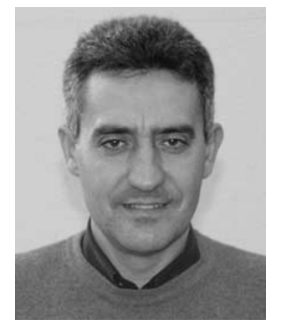

Pablo Gonzalez de Santos received the Ph.D. degree from the University of Valladolid, Valladolid, Spain.

$\mathrm{He}$ is a Research Scientist at the Spanish National Council for Research (CSIC), Madrid, Spain. Since 1981, he has been involved actively in the design and development of industrial robots and also in special robotic systems. His work during the last 15 years has been focused on walking machines. He worked on the AMBLER project as a Visiting Scientist at the Robotics Institute of Carnegie Mellon University, Pittsburgh, PA. Since then, he has been leading the development of several walking robot,s such as the RIMHO robot designed for intervention on hazardous environments, the ROWER walking machine developed for welding tasks in ship erection processes, and the SILO4 walking robot intended for educational and basic research purposes. He has also participated in the development of other legged robots, such as the REST climbing robot and the TRACMINER. He is now leading the DYLEMA project, which includes the construction of the SILO6 walking robot, to study how to apply walking machines to the field of humanitarian demining. 